\title{
AGB stars in the Magellanic Clouds
}

\section{The rate of star formation across the LMC}

\author{
M.-R. L. Cioni ${ }^{1}$, L. Girardi ${ }^{2}$, P. Marigo ${ }^{3}$, and H. J. Habing ${ }^{4}$ \\ 1 SUPA, School of Physics, University of Edinburgh, IfA, Blackford Hill, Edinburgh EH9 3HJ, UK \\ e-mail:mrc@roe.ac.uk \\ 2 Osservatorio Astronomico di Trieste, INAF, via G. B. Tiepolo 11, 34131 Trieste, Italy \\ 3 Dipartimento di Astronomia, Università di Padova, Vicolo dell'Osservatorio 2, 35122 Padova, Italy \\ 4 Sterrewacht Leiden, Niels Bohrweg 2, 2333 RA Leiden, The Netherlands
}

Received 27 July 2005 / Accepted 29 September 2005

\begin{abstract}
This article compares the distribution of $K_{\mathrm{s}}$ magnitudes of Large Magellanic Cloud (LMC) asymptotic giant branch (AGB) stars obtained from the DENIS and 2MASS data with theoretical distributions. These have been constructed using up-to-date stellar evolution calculations for low and intermediate-mass stars, and in particular for thermally pulsing AGB stars. A fit of the magnitude distribution of both carbon- and oxygen-rich AGB stars allowed us to constrain the metallicity distribution across the LMC and its star formation rate (SFR). The LMC stellar population is found to be on average 5-6 Gyr old and is consistent with a mean metallicity corresponding to $Z=0.006$. These values may however be affected by systematic errors in the underlying stellar models, and by the limited exploration of the possible SFR histories. Instead our method should be particularly useful for detecting variations in the mean metallicity and SFR across the LMC disk. There are well defined regions where both the metallicity and the mean-age of the underlying stellar population span the whole range of grid parameters. The $\mathrm{C} / \mathrm{M}$ ratio discussed in Paper I is a tracer of the metallicity distribution if the underlying stellar population is older than about a few Gyr. A similar study across the Small Magellanic Cloud is given in Paper III of this series.
\end{abstract}

Key words. galaxies: Magellanic Clouds - stars: late-type - Galaxy: abundances - stars: luminosity function, mass function

\section{Introduction}

Asymptotic Giant Branch (AGB) stars are among the brightest infrared members of a galaxy. They are useful indicators of galactic structure because they are widely distributed and easily noticed, and the ratio between carbon-rich (C-rich or C-type) and oxygen-rich (O-rich or M-type) AGB stars is an indicator of metallicity. Cioni \& Habing (2003) - Paper I - showed that this ratio varies over the face of the Large Magellanic Cloud (LMC) and concluded that a metallicity gradient is present in this galaxy; within the Small Magellanic Cloud (SMC) there are variations in this ratio but there is no clear pattern. Because AGB stars trace the stellar populations from $\sim 0.1$ to several Gyr, we want to constrain the global star formation rate (SFR) of the Magellanic Clouds, interpreting the large-scale magnitude distribution of AGB stars in the $K_{\mathrm{s}}$ band with the help of up-to-date stellar models. This work focuses on the stellar population in the field; we do not discuss clusters and associations. Although almost all previous work, a summary of which is given below, has been based on information from small regions within each galaxy, they more or less agree on the time-scale of major star formation events but it is not yet clear what these events were and what caused them.
Section 2 describes our selection of the sample of AGB stars from the DENIS catalogue towards the Magellanic Clouds and from the 2MASS catalogue. Section 3 describes the theoretical models used to construct a magnitude distribution while Sect. 4 compares the observed and theoretical distributions. Section 5 contains a comparison of our results with the information known from the literature while Sect. 6 concludes this paper. A similar study of the star formation rate in the SMC is presented in Paper III of the series.

\subsection{SFR in the $L M C-$ Review}

The LMC is a disk galaxy within which a bar is embedded. The disk, elongated toward the Galactic center, perhaps because of the tidal force induced by the Milky Way, is elliptical with ellipticity $\epsilon=0.199 \pm 0.008$; it is viewed at an inclination angle $i=34^{\circ} .7 \pm 6.2$ and a position angle of the major axis $\mathrm{PA}_{\text {maj }}=189.3 \pm 1.4$ (van der Marel 2001; van der Marel \& Cioni 2001). The off-center bar is about $3^{\circ}$ long, it is inclined at $15^{\circ} .1 \pm 2^{\circ} .7$ such that the eastern side is closer to us than the western side (Subramaniam 2004). The LMC thickness 
(about $2.4 \mathrm{kpc}$, Lah et al. 2005) is almost negligible compared to its distance.

The majority of the LMC globular cluster population is about 3-4 Gyr old and perhaps results from a tidal interaction with the SMC (van den Bergh 1999; Bekki et al. 2004). A few globular clusters formed before 11.5 Gyr ago and during recent distinct episodes of cluster formation (Hodge 1973; Subramaniam 2004, and references therein). Most star clusters dissolve in the LMC after about 1 Gyr (Hodge 1988) and then contribute to a stellar population in the field that is of varying age and metallicity. A decoupling between the rate of cluster formation and the SFR suggests that clusters may not be good tracers of the star formation in general (van den Bergh 1998).

Pioneering studies of different types of stars (Tifft \& Snell 1971) and of their distribution as a function of age (Isserstedt 1984) lead us to discover that the bulk of the LMC disk stellar population formed 3-5 Gyr ago (Butcher 1977). Some M giants (mostly AGB stars) as well C-rich AGB stars in the centre of the bar formed during this burst and others during another episode about 0.1 Gyr ago (Frogel \& Blanco 1983; Wood et al. 1985 - Fig. 2). This latter episode, possibly associated with a close encounter with the SMC, has produced a burst of star formation in both galaxies. It is supported by the distribution of early-type stars (Kontizas et al. 2004) and has been confirmed by the MACHO project comparing stellar evolution and pulsation models of Cepheids with their observed period-frequency distribution (Alcock et al. 1999). The same study also suggests that during the last $0.1 \mathrm{Gyr}$ star formation has been propagating from the South-East (SE) to the North-West (NW) along the bar where it is still ongoing (Hardy et al. 1984, Fig. 2). The formation of supergiant (massive) stars occurred more recently, about 7.5 Myr ago (Ardeberg 1976; Feitzinger et al. 1981).

Olson \& Pefia (1976) postulated a constant birthrate interrupted by periods of higher than average star formation. However, Rocca-Volmerange et al. (1981), by comparing integrated colours of the Magellanic Clouds with an evolutionary model, concluded that the evolution has been smooth, with a steady or slightly decreasing SFR in the past. Significant variations in the bolometric luminosity of AGB stars point to differences in the star formation history across the field (Reid \& Mould 1984, Fig. 2). In particular carbon stars at the periphery might be younger (Costa \& Frogel 1996, Fig. 2).

Deep $B$ and $V$ band observations were compared by Bertelli et al. (1992, Fig. 2) and by Vallenari et al. (1996a,b, Fig. 2) with synthetic colour-magnitude-diagrams and luminosity functions (LFs). The authors concluded that from region to region star formation took place at different epochs. In particular, the SFR in some fields was low until $4 \pm 0.5 \mathrm{Gyr}$ ago, but this burst would occur about 2 Gyr later if the models include overshooting; the SFR was enhanced 6-8 Gyr ago in the region East (E) of the bar while in the region West (W) of the center there was star formation 2-3 Gyr ago. About $8^{\circ}$ from the LMC center Gallart et al. (2004, Fig. 2) using high quality $V$ and $I$ photometry derived an intermediate age $(>2.5 \mathrm{Gyr}$ old $)$ stellar population as well as a younger $(\approx 1.5 \mathrm{Gyr}$ old $)$ component which is perhaps the result of an LMC-SMC encounter or from a merger of the LMC with a smaller galaxy. Although there is an indication of an age gradient, in the sense: "older stars towards the periphery" ( $>8$ Gyr old), there is only a small population as old and metal poor as that of the Milky Way halo globular clusters and dwarf spheroidal galaxies (see also Stryker 1984).

Observations with the Hubble Space Telescope (HST) in the LMC outer disk revealed for the first time the oldest mainsequence turnoff point (Gallagher et al. 1996, Fig. 2). A best fit model of the main-sequence suggests a roughly constant SFR over $10 \mathrm{Gyr}$ with an increase around 2 Gyr (Holtzman et al. 1997; Geha et al. 1998 - Fig. 2) although in the vicinity of the globular cluster NGC 1866 an increase in the star formation rate probably occurred a Gyr earlier (Stappers et al. 1997, Fig. 2). A relatively larger component of older stars around globular clusters was also found by Olsen (1999, Fig. 2). In the inner disk a burst at about 1 Gyr may correspond to the formation of the bar (Elson et al. 1997, Fig. 2) but an older bar (4-6 Gyr) was claimed by Smecker-Hane et al. (1999, Fig. 2). Differences in either age or metallicity were detected between this inner disk field and the outer disk field studied by Gallagher et al. (1996). Near the bar Javiel et al. (2005, Fig. 2) confirm a stars forming event at $1 \mathrm{Gyr}$ and one before $10 \mathrm{Gyr}$ (see also Holtzman et al. 1999, Fig. 2) with a clear gap from 3-6 Gyr. In the centre of the bar Ardeberg et al. (1997, Fig. 2) identified two strong populations: a young component that originated about 0.5 Gyr ago and an older component that originated between 2 and 9 Gyr ago.

Thus, information about the star formation history of the LMC has been obtained from the study of many relatively small regions located in the outer and inner disk as well as along the bar. There are regions of the galaxy that can be described by a relatively uniform SFR across several Gyr. The dominant stellar population is of intermediate-age $(>2.5 \mathrm{Gyr})$ and extends to the remote periphery. A burst of star formation has occurred between 1 and $3 \mathrm{Gyr}$; although some authors attribute to this event the formation of the bar, there is evidence that stars as old as 4-8 Gyr exist in the bar as well as in the disk. Only a detailed kinematic study of the stellar population will reveal its distinct components. This as well as more recent bursts of star formation are probably due to a close passage with the Mikly Way and the SMC. In the outer disk, searches for stars similar to those in the halo of the Milky Way have shown that metal poor old giants are lacking. AGB stars formed essentially during two major epochs: around $10^{8} \mathrm{yr}$ ago for the most massive and a few Gyr ago for lower masses. Globular clusters are older than about $11 \mathrm{Gyr}$ or younger than about $4 \mathrm{Gyr}$.

\section{Observational sample of AGB stars}

\subsection{Selection of $A G B$ stars from $D C M C$}

The DENIS catalogue towards the Magellanic Clouds (DCMC - Cioni et al. 2000a) provides simultaneous observations in $I J K_{\mathrm{s}}$ of over one million point sources in the direction of the LMC. Sources detected in all three wavebands have been used in Cioni \& Habing (2003, Paper I) to study the metallicity distribution across the surface of the galaxy.

Following the same criteria as in Paper I we selected an almost complete sample of AGB stars. In the colour-magnitude diagram $(I-J, I)$ the AGB stars, irrespective of their chemical 
nature, lie in the region of objects brighter than the tip of the red giant branch (TRGB - Cioni et al. 2000c) and redder than a diagonal line that distinguishes them from younger stars or foreground stars (see Fig. 1 of Cioni et al. 2000b). Our sample includes O-rich AGB stars of early $\mathrm{M}$ spectral sub-type (i.e. M 0-M 1), close to the TRGB, but excludes AGB stars with thick circumstellar envelopes that are fainter than the TRGB location ${ }^{1}$. The spectral sub-type of O-rich AGB stars is a function of their $(I-J)$ colour (Fluks et al. 1994; Blanco et al. 1980).

The TRGB is at $I=14.54$ and to account for the extinction along the line of sight we adopt an average extinction ${ }^{2}$ corresponding to $E(B-V)=0.15$ as given by Westerlund (1997). Using the extinction law of Glass (1999) we obtain the following absorptions: $A_{I}=0.27, A_{J}=0.11$ and $A_{K_{\mathrm{s}}}=0.04$. Before selection of the AGB population the data have been dereddened using the extinction values listed above. Because we use both DCMC and 2MASS data (see below) the DCMC $J K_{\mathrm{S}}$ data have been transformed into the 2MASS photometry by applying the systematic shifts derived by Delmotte et al. (2002): $J^{2 \mathrm{MASS}}=J^{\mathrm{DCMC}}+0.11$ and $K_{\mathrm{s}}^{2 \mathrm{MASS}}=K_{\mathrm{s}}^{\mathrm{DCMC}}+0.14$. A diagonal line separates AGB stars from younger and foreground objects:

$I_{0}=-4.64 \times\left(I-J^{2 \mathrm{MASS}}\right)_{0}+19.78$,

where the subscript 0 indicates that magnitudes and colours are corrected for extinction. These selection criteria result in 32801 candidate AGB stars (Fig. 1).

\subsection{Selection of AGB stars from $2 M A S S$}

The 2MASS all sky survey (Skrutskie 1998) provides simultanous $J H K_{\mathrm{s}}$ observations of sources distributed in the whole sky as well as in the direction of the Magellanic Clouds. In the $\left(J-K_{\mathrm{s}}, K_{\mathrm{s}}\right)$ colour-magnitude-diagram most AGB stars are brighter than the TRGB and are redder than younger and foreground objects. There are some AGB stars as faint as the TRGB itself or slightly fainter and they cannot be distinguished from evolved red giant branch (RGB) stars by near-infrared photometric criteria. These stars are usually O-rich and of early spectral subtype (i.e. M 0-M 1).

After dereddening the data using the absorption values derived in the previous sub-section, candidate AGB stars occupy the region of the colour magnitude diagram $\left(J-K_{\mathrm{s}}, K_{\mathrm{s}}\right)$ enclosed by two lines:

$K_{\mathrm{s} 0}=-0.48 \times\left(J-K_{\mathrm{s}}\right)_{0}+13$

$K_{\mathrm{s} 0}=-13.333 \times\left(J-K_{\mathrm{s}}\right)_{0}+24.666$.

A discussion about the precise location of these lines is given in Sect. 3.3. Figure 1 shows the location of these lines as well a third line to distinguish between O-rich and C-rich AGB stars presented in the next sub-section.

${ }^{1}$ AGB stars with thick circumstellar envelopes are expected to be just a small fraction $(<10 \%)$ of the total number of AGB stars. This upper limit has been estimated from the number of sources with $\left(J-K_{\mathrm{s}}\right)>2.5$.

${ }^{2}$ Differential reddening has a negligible effect in the $K_{\mathrm{s}}$ band.

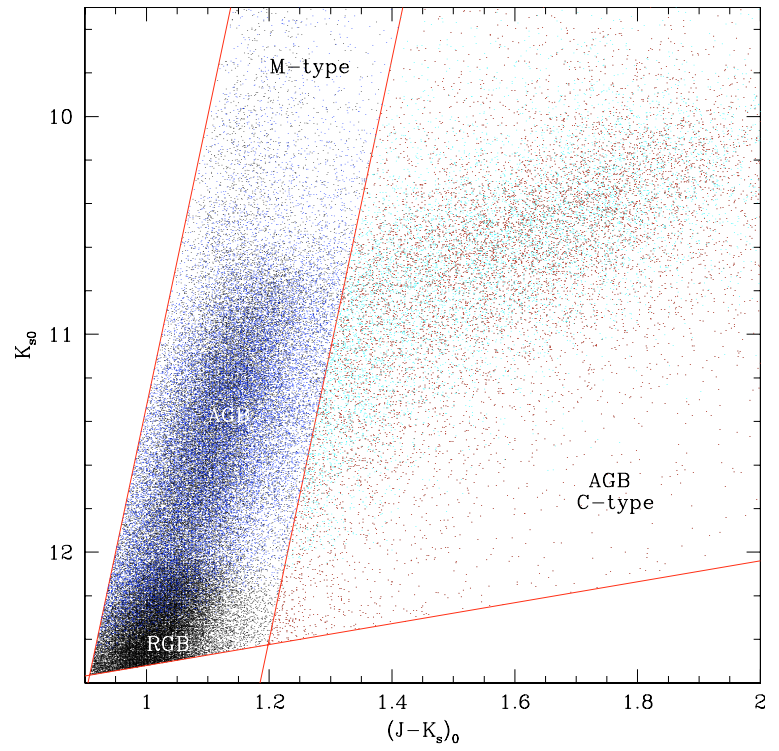

Fig. 1. Distribution of candidate LMC AGB stars in the colour magnitude diagram $\left(J-K_{\mathrm{s}}, K_{\mathrm{s}}\right)$. Because of the large amount of data this figure can only be appreciated in colour. Stars selected from the 2MASS data are shown in black (M-type) and red (C-type) while stars selected from the DCMC data are shown in blue (M-type) and cyan (C-type). Note that 2MASS selected sources most probably include genuine RGB stars at the faintest magnitudes of M-type candidates as well as more stars than selected from the DCMC data distributed overall. This is probably a result of the different selection criteria as well as the different photometric accuracy.

In summary 61078 candidate AGB stars were selected in the LMC. We included only sources detected in all three photometric wavebands and with good photometry and accurate positions (this is equivalent to selecting stars with 2MASS rd_flg $=1,2$ or 3 in each wave band), to strengthen the reliability of the sample (Fig. 1). These stars occupy the same surface area as DCMC stars and in particular were extracted from the all sky 2MASS data release as those included in a polygon: $61.61^{\circ}<\alpha<101.79^{\circ}$ and $-61^{\circ}<\delta<-77^{\circ}$.

\subsection{Photometric selection of $M$ and $C$ stars}

AGB stars can be either O-rich or C-rich. The distinction between spectral types can be made because different molecules dominate the stellar spectra. O-rich stars are essentially dominated by $\mathrm{VO}, \mathrm{TiO}$ and $\mathrm{H}_{2} \mathrm{O}$ molecules while $\mathrm{CN}$ and $\mathrm{C}_{2}$ molecules dominate the spectra of C-rich AGB stars. O-rich AGB stars have an approximately constant $\left(J-K_{\mathrm{S}}\right)$ colour and span a large range of $K_{\mathrm{S}}$ magnitudes. C-rich AGB stars have redder colours and are constrained to a smaller magnitude interval. However there is a range of colours populated by both O-rich and C-rich AGB stars (Groenewegen 2004).

We discriminate the two spectral classes of AGB stars using a line at

$K_{\mathrm{s} 0}=-13.333 \times\left(J-K_{\mathrm{s}}\right)_{0}+28.4$,

which is also shown in Fig. 1. In Cioni \& Habing (2003) we use spectroscopically confirmed AGB stars and theoretical models 
to discriminte between O-rich and C-rich AGB stars using a vertical line at $J-K_{\mathrm{s}}=1.4$. Here we improve the discrimination criteria using a diagonal line with the same slope as the line that discriminates AGB stars from younger and foreground stars that also agrees with the slope of the O-rich AGB branch. C-rich AGB stars depart from this branch towards redder colours under the effect of molecular blanketing.

The use of sharp boundaries to select a group of stars may be affected by "migration" of objects across the boundaries because of uncertainties due to photometric errors or to variability. The errors are small ( $<0.02 \mathrm{mag})$, but the "migration" due to variability may be significant. Most AGB stars are classified as long-period variables and vary over several magnitudes in the visual with a periodicity of a few hundred days and a more or less regular light-curve shape (e.g. Cioni et al. 2001, 2003). At near-infrared wavelengths the amplitude of the variation is below 1 magnitude and the variation in the $J-K_{\mathrm{s}}$ colour is least a factor of ten smaller. However observations at a single epoch, like those available for this paper, caught stars at a specific position in the light curve, some close to minimum brightness, others close to maximum brightness and some in the middle. Because of these differences both magnitude and colour will be affected by random and not systematic shifts considerably reducing the effect of variability.

Age and metallicity also contribute to the location of individual AGB stars in the near-infrared colour-magnitude diagram. Cioni \& Habing (2003) have shown that the ratio between $\mathrm{C}$-rich and O-rich $\mathrm{AGB}$ stars (the $\mathrm{C} / \mathrm{M}$ ratio) indicates a variation $\Delta[\mathrm{Fe} / \mathrm{H}]=0.75 \mathrm{dex}$ within the LMC. This covers a range of metallicities from the SMC to the Milky Way and corresponds to a variation in the position of the TRGB of \pm 0.2 mag for a stellar population 3-10 Gyr old. We assume that AGB stars above the TRGB will be affected by a similar shift in $K_{\mathrm{s}}$ magnitude (Glass et al. 2004). Similarly $J-K_{\mathrm{s}}$ varies by \pm 0.15 mag.

In this paper we assume that within a small region $\left(0.16 \mathrm{deg}^{2}\right.$; Sect. 4$)$ age and metallicity are constant (i.e. stars formed at the same time in a homogeneous chemical environment) and we study differences between these regions. While variability affects all AGB stars regardless of metallicity, an error in the choice of the diagonal line that divides O-rich from C-rich AGB stars may systematically affect the interpretation of the number of stars in each group. This effect can be quantified as follows: within $\Delta\left(J-K_{\mathrm{s}}\right)=0.15 \mathrm{mag}$ of the line there is a number of stars equivalent to $5 \%$ of the total number of AGB candidates; these cover about 10 bins of $\Delta K_{\mathrm{s}}=0.2 \mathrm{mag}$ each. In a sample region $\left(0.16 \mathrm{deg}^{2}\right)$ this corresponds to misplacing, on average, not more than 1 star per bin (Fig. 3). We conclude that only where too few stars occupy a single bin our conclusions may be uncertain.

\subsection{Subdivision of the $L M C$ area}

In order to study the shape of the magnitude distributions as a function of spatial coordinates we divided the LMC area into rings and sectors: six concentric rings centered at $\alpha=82.25^{\circ}$ and $\delta=-69.5^{\circ}$ at a radius of $1.4^{\circ}, 2.5^{\circ}, 3.4^{\circ}, 4.4^{\circ}, 5.4^{\circ}$

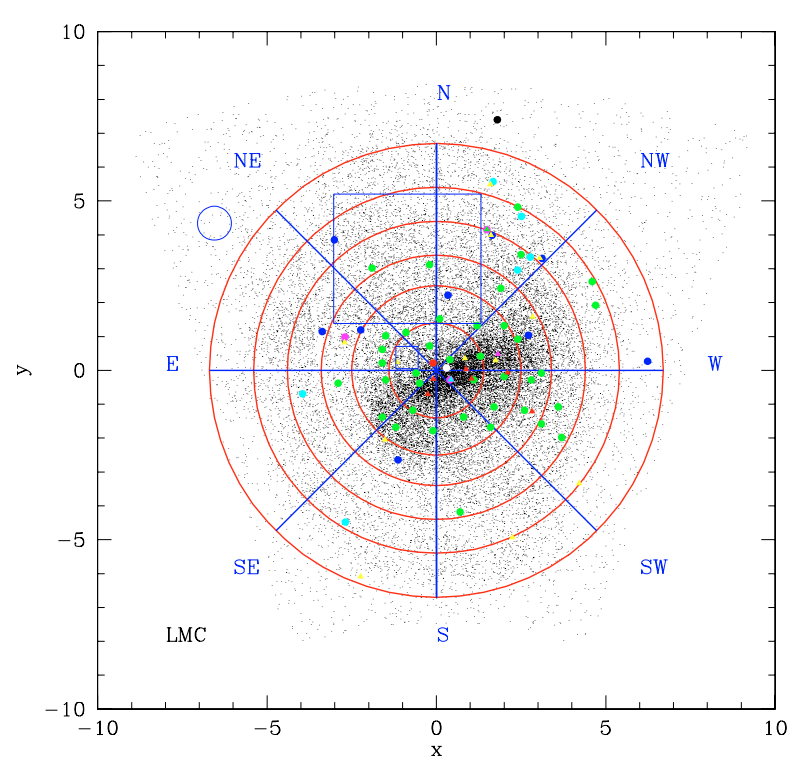

Fig. 2. Distribution of the AGB stars in the LMC. Data are from the DCMC and include only sources detected in three DENIS wave bands. The division into rings and sectors used in this article is indicated. The center is at $\alpha=82.25^{\circ}$ and $\delta=-69.5^{\circ}$. Coloured symbols indicate the location of regions analysed by other authors, in particular: thick dots for Costa \& Frogel (1996, green), Javiel et al. (2005; cyan), Vallenari et al. (1996b, blue), Holtzman et al. (1999, magenta), Gallart et al. (2004, black), Elson et al. (1997, white), Ardeberg et al. (1997, red) and triangles for Hodge (1988, yellow), Olsen et al. (1999, red), Gallagher et al. (1996), Holtzman et al. (1997) and Geha et al. (1998) (green), Tifft \& Snell (1971, magenta), Smecker-Hane et al. (1999, cyan). The NGC 1866 cluster corresponds to a concentration of four different symbols (N-NW) while large empty regions refer to studies by: Reid \& Mould (1984, big rectangle), Stryker (1984, circle) and Wood et al. (1985, small square).

and $6.7^{\circ}$, respectively, as well as eight sectors at an angular aperture of $45^{\circ}$. Rings are numbered $0-5$ with increasing radius and sectors by compass direction (E, NE, N, NW, W, SW, $\mathrm{S}, \mathrm{SE}$ ); see Fig. 2. The outer four rings coincide with the division in van der Marel \& Cioni (2001); see also Paper I, while their innermost region has been split in two.

\subsection{Comparison between DCMC and 2MASS samples}

The important advantage of having simultaneous $I J K_{\mathrm{s}}$ band data is that it is possible to include O-rich AGB stars of early spectral type in the selected AGB sample. $J K_{\text {s }}$ photometry does not contain enough information for this task because while all AGB stars are located above the TRGB in the $I$ band, some will be below the TRGB in the $K_{\mathrm{s}}$ band, therefore they are mixed with RGB stars. This is clearly demonstrated in Fig. 1.

On the other hand the 2MASS photometry is somewhat more precise than the DENIS photometry and 2MASS observations reach about 1 mag fainter objects. The important consequence is that the sequences occupied by different stars in the colour-magnitude diagram are narrower and better defined.

A comparison of DCMC and 2MASS selected AGB stars allow us to: (i) test the contamination of the sample of 
AGB stars by RGB stars of early spectral subtype; and (ii) determine the completeness of AGB stars above the TRGB in the $K_{\mathrm{s}}$ band that should be equally recovered using both photometric selection criteria in both datasets.

The AGB stars are selected in the $(I-J, I)$ diagram from DCMC-data as described in Sect. 2.1. The same stars then are plotted in the $\left(J-K_{\mathrm{s}}, K_{\mathrm{s}}\right)$ diagram and it is this latter distribution that has lead us to define three lines to characterize the region that all O-rich and C-rich AGB stars occupy.

Let us focus on a particular ring (number 3 ) and sector $(\mathrm{N})$. Figure 3 shows the distribution of O-rich and C-rich stars selected from each data set as well as a combined histogram produced as follows. At magnitudes above the TRGB $\left(K_{\mathrm{s}}<\right.$ 12) a simple average was calculated of stars selected from 2MASS and DCMC. This is justified because at these magnitudes both data sets should have detected the same AGB star candidates irrespective of their $I$ or $H$ magnitude. Therefore we attribute differences to a different photometric quality, sensitivity and possibly different arrays and flags of image or reduction origin that may have lead to exclusion of some detections, even at these relatively bright magnitudes. Averaging measurements from both catalogues has the important advantage of reducing migration effects between adjacent bins and smoothning the effect of variability. 2MASS and DCMC observations were obtained on average from a few to several months apart and their mean magnitudes should closely resemble the average magnitude that one would obtain if a near-infrared light curve were available for each source. The distributions of the C stars from DCMC and from 2MASS are very similar. This is also the case in other sectors whether they contain more or fewer stars. A Kolmogorov-Smirnov test indicates a probability of about $80-90 \%$ that both C-star distributions originate from the same population.

DCMC and 2MASS samples also agree when one considers the O-rich stars brighter than $K_{\mathrm{s}}=12 \mathrm{mag}$. At $K_{\mathrm{s}}>12$ the distribution of O-rich stars extracted from the 2MASS sample departs considerably from that of the stars extracted from the DCMC sample and we attribute this to the strong contamination of the AGB sample by RGB stars in the 2MASS data. Simply averaging the DCMC and 2MASS samples will produce erroneous results, namely a too large number of candidate AGB stars of early spectral sub-type. Because of the availability of the I-DCMC measurement we are confident that we avoided most of these RGB/pseudo-AGB stars and we conclude that the best distribution at $K_{\mathrm{s}}>12$ is given by DCMC stars only. Note that at these magnitudes amplitudes of variability decrease while photometric errors increase; it is likely that the effect of migration between adjacent bins becomes more important than the effect of variability.

\section{Theoretical models}

\subsection{General description}

The $J K_{\mathrm{s}}$-band photometry of the Large Magellanic Cloud has been simulated using a population synthesis code called TRILEGAL (Girardi et al. 2005) that randomly generates a population of stars following a given SFR, age-metallicity

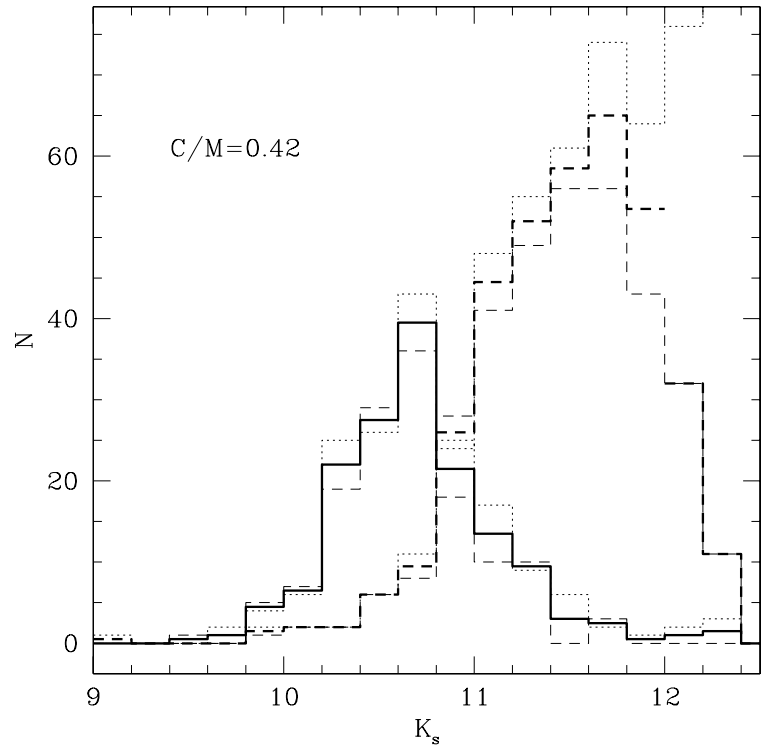

Fig. 3. Distribution of $\mathrm{C}$ and $\mathrm{M}$ stars as a function of $K_{\mathrm{s}}$ mag in sector $\mathrm{N}$ of ring number 3 (Fig. 2). The histogram peaking at about $K_{\mathrm{s}}=10.7$ is due to $\mathrm{C}$ stars while $\mathrm{M}$ stars populate the peak at fainter mag $\left(K_{\mathrm{s}}=11.7\right)$. The dotted line indicates the distribution of stars selected from the 2MASS data while the dashed line indicates the distribution of stars selected from the DCMC data. The thick continuous and dashed lines are the average of both data sets for $K_{\mathrm{s}}<12$ while for $K_{\mathrm{s}}>12$ the distribution of M stars is strictly that of DCMC data. These thick lines show the final histogram obtained from the observational data that is later compared with a theoretical distribution. The ratio between $\mathrm{C}$ and $\mathrm{M}$ stars obtained from this final histogram is also indicated.

relation (AMR) and initial mass function (IMF). The stellar intrinsic properties (luminosity $L$, effective temperature $T_{\text {eff }}$, surface gravity $g$, etc.) are interpolated over a large grid of stellar evolutionary tracks, based on Bertelli et al. (1994) for massive stars, Girardi et al. (2000) for low- and intermediatemass stars, and complemented with grids of thermal pulsing AGB (TP-AGB) tracks calculated by means of Marigo et al.'s (1999) synthetic code. This latter code has been improved as follows.

First, the TP-AGB computations now adopt lowtemperature opacities consistent with the actual chemical composition of the stellar envelope, rather than for the (usually assumed) solar-scaled compositions. As first shown by Marigo (2002) this choice has dramatic consequences for the evolution of TP-AGB stars, that include significant changes in their $T_{\text {eff }}$ and lifetimes, providing a natural explanation for the appearance of a red tail of $\mathrm{C}$ stars in colour-magnitude diagrams (CMDs) involving the $\left(J-K_{\mathrm{s}}\right)$ colour (see Marigo et al. 2003).

Second, the TP-AGB tracks adopt a new formalism to describe the efficiency of the third dredge-up process and the mass loss rates (Marigo et al., in preparation). The main novelties, with respect to the formalism described in Marigo et al. (1999), are: the ratio between the mass of dredged-up material and the total core mass growth at each interpulse phase, $\lambda$, is a function of mass and $Z$ according to the Izzard et al. (2004) stellar models. As in Marigo et al. (1999) the criterion for 
activating the third dredge up is based on the minimum temperature reached at the basis of the convective envelope after shell He-flash episodes, $T_{\mathrm{b}}^{\mathrm{dred}}$; this parameter, however, now includes a small metallicity dependence as suggested by the calibration of the carbon star luminosities in the Magellanic Clouds. The mass loss rates are still described using the Vassiliadis \& Wood (1993) prescription, but now using the period values given by either fundamental mode or first overtone. The transition between these two pulsation modes is predicted according to a series of theoretical indications from pulsation models (Ostlie $\&$ Cox 1986). The details of these TP-AGB models will be given in a forthcoming paper. They provide a reasonable reproduction of the C star LFs in both Magellanic Clouds, and of the lifetime of the carbon phase as a function of mass as deduced from C-star counts in LMC star clusters (see Girardi \& Marigo 2002).

In this paper we use complete grids of tracks for 5 different metallicities, namely $Z=0.0004,0.001,0.004,0.008$ and 0.019 . The $Z=0.004,0.008$ and 0.019 cases are close to the metallicity values found in young populations of the SMC, LMC and Solar Neighbourhood, respectively. Interpolations among these grids allow us to generate stars for any intermediate metallicity between $Z=0.0004$ and 0.019 .

The evolution along the TP-AGB phase is initially described in terms of the properties in the quiescent stages between thermal pulses. We then include in the simulations the effect of luminosity variations driven by thermal pulses, in the same way as Marigo et al. (2003). This produces a broadening - especially to lower luminosities and high $T_{\text {eff }}-$ of the distribution of TP-AGB stars in the HR diagram.

\subsection{Construction of the theoretical $K_{s}$-band distribution}

Once the intrinsic stellar properties $\left(L, T_{\text {eff }}, \mathrm{C} / \mathrm{O}\right)$ have been determined, we simulate the photometry by applying the extended tables of bolometric corrections (BCs) from Girardi et al. (2002) for O-rich stars (with $\mathrm{C} / \mathrm{O}<1$ ), and by empirical relations for $\mathrm{C}$-rich stars (with $\mathrm{C} / \mathrm{O}>1$ ).

The BCs for M-type stars are based on the empirical spectra and $T_{\text {eff }}$-colour scale by Fluks et al. (1994). For other O-rich stars with $T_{\text {eff }} \gtrsim 4000 \mathrm{~K}$, instead, they are entirely based on ATLAS9 model atmospheres (see Kurucz 1992; Castelli et al. 1997). Since at LMC metallicities the M-type fraction of the AGB phase develops mostly at $T_{\text {eff }}$ lower than $4000 \mathrm{~K}$, the Fluks et al. (1994) set of corrections is actually the most relevant for the present work. The magnitudes and colours we simulate in this way correspond to the DENIS $I J K_{\mathrm{s}}$ and to the 2MASS $J H K_{\mathrm{s}}$ filters.

Regarding C-type stars, we rely on a few empirical relations. The $\mathrm{BC}$ in the $K$ band is taken from the relation by Costa \& Frogel (1996). The $(J-K)$ colour is derived from the $T_{\text {eff }}-(J-K)-\mathrm{C} / \mathrm{O}$ relation from Marigo et al. (2003), which itself is based on a fit to the empirical data by Bergeat et al. $(2001)^{3}$.

\footnotetext{
3 The fitting relations to BC in the $K$-band (Costa \& Frogel 1996, show a dispersion of just 0.05 mag, which is comparable to the
}

Finally, we simulate the photometric errors in the following way: for each star of $J$ and $K_{\mathrm{s}}$ we compute $\sigma_{J}$ and $\sigma_{K_{\mathrm{s}}}$ using the fitting formulas by Bonatto et al. (2005, and private communication). Then, the single errors are randomly generated from Gaussian distributions of widths $\sigma_{J}$ and $\sigma_{K_{\mathrm{s}}}$, and added to $J$ and $K_{\mathrm{s}}$. This helps in producing clean-looking CMDs, but is not a critical step since at the magnitudes involved in this study, the photometric errors are typically very low (less than $0.03 \mathrm{mag}$ ).

\subsection{Main features and behaviour of the theoretical distributions}

Before the comparison with observational data, we discuss the basic behaviour of present models, as a function of galactic properties. The main parameters describing a galaxy are the SFR, $\psi(t)$, and the mean metallicity $Z(t)$, as a function of stellar age $^{4} t$.

Of course, we can test only a limited number of all possible SFR and AMR functions. We will use existing, and hopefully realistic models for these functions for the LMC. However, we found it useful to begin with simple functions that may be somewhat unrealistic but very instructive.

\subsubsection{Varying the metallicity at a constant SFR}

In this case, $\psi(t)=\psi_{0}$, where $\psi_{0}$ is an arbitrary constant chosen so as to give a reasonable number (several thousand) of AGB stars. $Z$ is also kept at a constant value, $Z(t)=Z_{0}$, inside each simulation. We then analyse the effect of varying $Z_{0}$ between our limiting values of 0.0004 and 0.019 .

A sequence of such models is illustrated in Fig. 4. They show some remarkable changes in the appearance and location of the several CMD features, that will be commented on later in this work.

The continuous lines connecting the squares in Fig. 5 illustrate the variation of $\mathrm{C} / \mathrm{M}$ with metallicity $[\mathrm{M} / \mathrm{H}]=$ $\log \left(Z_{0} / Z_{\odot}\right)$, for two different selection criteria of $\mathrm{C}$ and $\mathrm{M}$ stars. The most significant of these criteria is the model $C / M$ ratio, that we obtain by simply counting the simulated TP-AGB stars of different $\mathrm{C} / \mathrm{O}$ ratio $(\mathrm{C} / \mathrm{O}<1$ for $\mathrm{M}$-type, $\mathrm{C} / \mathrm{O}>1$ for $\mathrm{C}$ type). Then we have the photometric $C / M$ ratio, defined in the following way: first, we draw three different lines in the CMD, namely:

$$
\begin{aligned}
& K_{0}=-0.48\left(J-K_{\mathrm{s}}\right)+13.022+0.056[\mathrm{M} / \mathrm{H}] \\
& K_{1}=-13.333\left(J-K_{\mathrm{s}}\right)+25.293+1.568[\mathrm{M} / \mathrm{H}] \\
& K_{2}=-13.333\left(J-K_{\mathrm{s}}\right)+29.026+1.568[\mathrm{M} / \mathrm{H}]
\end{aligned}
$$

C stars are defined by $K<K_{0}$ and $K>K_{2}$, M stars by $K<K_{0}$ and $K_{1}<K<K_{2}$. The significance of these three lines is clear when looking at Fig. 4. The photometric $\mathrm{C} / \mathrm{M}$ ratio is obtained

photometric errors in the observed samples). The possible errors in the attribution of $J-K$ will affect only the selection between $C$ - and M-type stars in the fainter end of their magnitude distribution, but not the bulk of the AGB stars.

${ }_{4}$ The IMF of stars is assumed to be independent of age and equal to the log-normal function of Chabrier (2001). None of the results of this paper depend on the detailed shape of the IMF. 

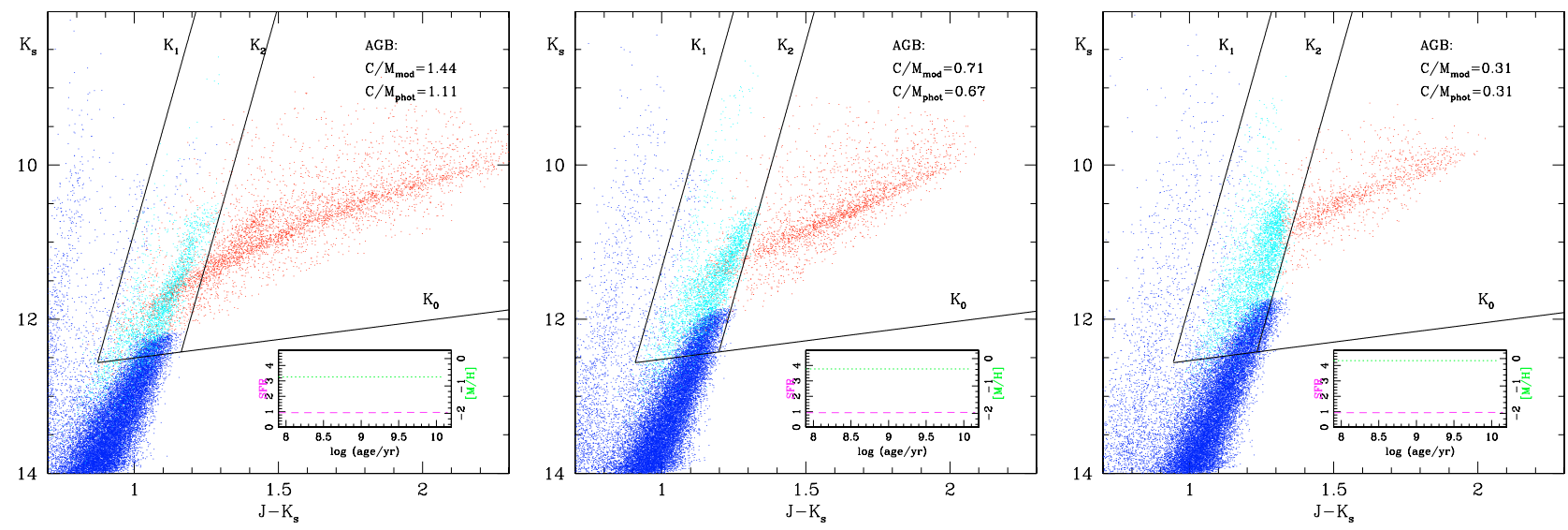

Fig. 4. Simulated CMDs for models that assume a constant SFR, and three different values of metallicity for stars of all ages: $Z=0.004$ (left panel), $Z=0.008$ (middle) and $Z=0.016$ (right). For comparison, the inserts illustrate the shape of SFR (dashed line, left axis) and AMR for each model (dotted line, right axis). A distance of $50 \mathrm{kpc}$ is assumed (this is approximately the distance to the LMC). The straight lines delimit regions of the CMD that were used in the photometric selection of C- and M-type stars (see text). In the electronic version of this paper only, different kinds of stars are denoted with different colours, namely blue for all stars before the TP-AGB phase, cyan for O-rich and red for C-rich TP-AGB stars. Notice the marked differences in the location and relative number of M and C stars. The TRGB is easily noticed at $K_{\mathrm{s}} \sim 12.3$, $\sim 12.0$ and $\sim 11.7$ in the left, middle and right panels, respectively.

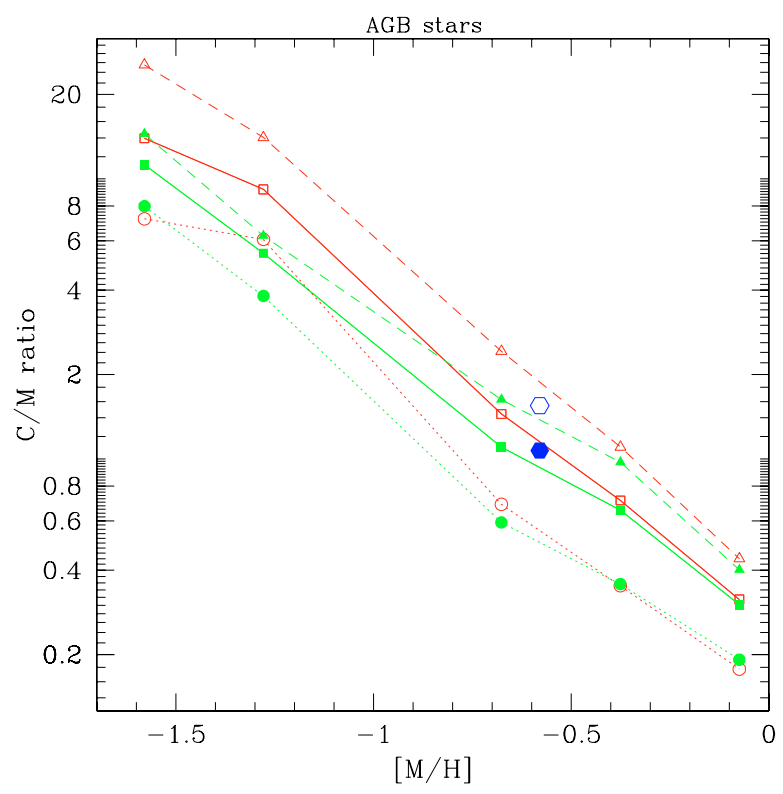

Fig. 5. Variation of $\mathrm{C} / \mathrm{M}$ with metallicity $[\mathrm{M} / \mathrm{H}]$ for three different cases of SFR - from top to bottom: $\propto \exp (-t / 5 \mathrm{Gyr})$ (triangles and dashed lines), constant (squares and continuous lines), and $\propto \exp (t / 5 \mathrm{Gyr})$ (circles and dotted lines) - and using both the model (empty symbols) and photometric (filled symbols) selection criteria for AGB stars above the TRGB. Notice that both criteria produce the same behaviour of $\mathrm{C} / \mathrm{M}$ as a function of $[\mathrm{M} / \mathrm{H}]$. The hexagons represent the C/M for a "realistic model" of the LMC (see Sect. 3.3.3).

by the division of the number of stars counted in these two different regions of the CMD. The lines $K_{1}$ and $K_{2}$ are parallel with a separation $\Delta\left(J-K_{\mathrm{s}}\right)=0.28 \mathrm{mag}$. This is wide enough to completely encompass the sequences of O-rich TP-AGB stars (especially at high metallicities where their colour distribution is broader), avoiding as much as possible the contamination by other kinds of stars. In these equations, the terms with $[\mathrm{M} / \mathrm{H}]$ reflect how the theoretical sequences shift with the metallicity assumed in the simulations.

Figure 5 shows that the photometric $\mathrm{C} / \mathrm{M}$ ratio as function of $[\mathrm{M} / \mathrm{H}]$ is parallel to the model $\mathrm{C} / \mathrm{M}$ ratio, in spite of the incomplete elimination of RGB stars from the AGB sample. Because the bluest $\mathrm{C}$-stars will be misclassified as M-type stars we will always have $\mathrm{C} / \mathrm{M}(\mathrm{phot})<\mathrm{C} / \mathrm{M}($ model $)$. Both $\mathrm{C} / \mathrm{M}$ values depend on $[\mathrm{M} / \mathrm{H}]$ and, for the case of constant SFR, have values typically between 0.25 and 14 .

\subsubsection{Varying the SFR at a constant metallicity}

As before we take $Z(t)=Z_{0}$, and keep it at the same value inside each simulation. What changes from case to case is the SFR. We adopt the following family of exponentially increasing/decreasing SFRs:

$\psi(t)=\psi_{0} \exp (t / \alpha)$

where $t$ is the look-back time (stellar age) and $\alpha$ is positive/negative for increasing/decreasing look-back time. In our calculations $\alpha$ will take values $2,5, \infty,-2,-5$. Again, $\psi_{0}$ is an arbitrary constant chosen so as to produce a reasonable number of stars.

A sequence of such models is illustrated in Fig. 6. The location of the important CMD features is the same in the three panels but the relative numbers of stars change as does the $\mathrm{C} / \mathrm{M}$ ratio. Figure 5 illustrates the variation of $\mathrm{C} / \mathrm{M}$ for a few SFR functions as a function of metallicity.

These simulations thus indicate that the $\mathrm{C} / \mathrm{M}$ ratio of TP-AGB stars depends strongly on the shape of SFR, a dependence that so far has not been much discussed. The top panel of Fig. 7 illustrates this effect. It presents a histogram of the ages of the stars in a sample generated under the assumption that the SFR and the metallicity both have been constant $(Z=0.008)$ over a period of $10 \mathrm{Gyr}$. The figure shows that the age distribution of $\mathrm{C}$ stars (red, continuous curve) has a somewhat marked 

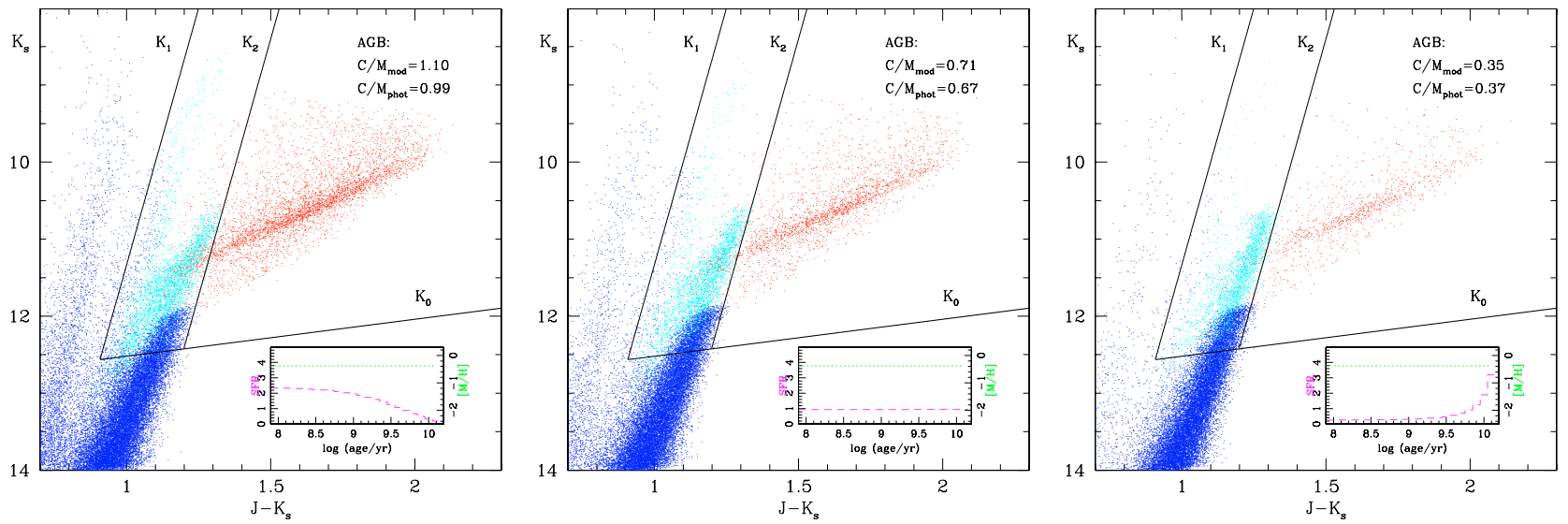

Fig. 6. Simulated CMDs for models with a constant metallicity, $Z_{0}=0.008$, and three different cases of varying SFR: decreasing $(\alpha=-5$ Gyr, left panel), constant (middle panel) and increasing ( $\alpha=+5 \mathrm{Gyr}$, right panel) with stellar age. The insert, straight lines and $\mathrm{C} / \mathrm{M}$ definitions are described in Fig. 4. Notice the large changes in relative numbers of different kinds of stars.

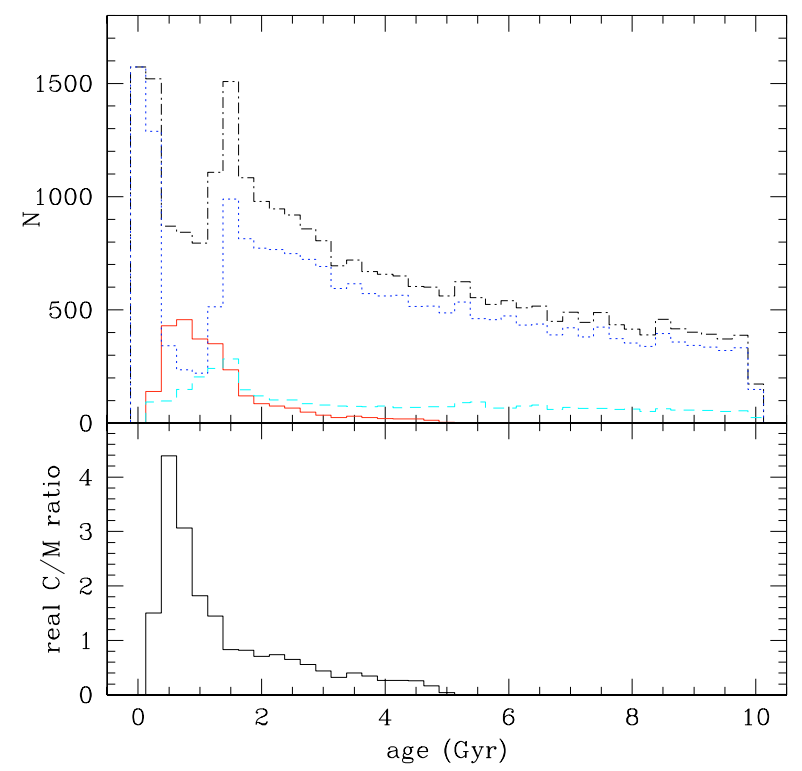

Fig. 7. Top panel: age distribution of different stars when the SFR and $Z=0.008$ have been constant over the last $10 \mathrm{Gyr}$. Only stars with $M_{K}<-4.5(K<14$ at the LMC distance) have been considered. The C-rich (red, continuous line) and O-rich (blue, dashed line) TP-AGB stars are in general less numerous than stars from previous evolutionary phases (blue, dotted lines; these are mostly coreHe burning and early-AGB stars for $t<1$ Gyr, and RGB stars for $t>1 \mathrm{Gyr})$. The blue, upper dot-dashed line indicates the total number of $K<14$ stars. Bottom panel: the C/M ratio of TP-AGB stars, as a function of stellar age, for the same model.

peak at $0.75 \mathrm{Gyr}$ and that there are no $\mathrm{C}$ stars younger than 0.25 Gyr and older than 5 Gyr. The M stars (blue, dashed) show a peak at an age of about $1.5 \mathrm{Gyr}$, but they have a much broader distribution to both younger and older ages. Thus, the $\mathrm{C} / \mathrm{M}$ ratio (bottom panel of Fig. 7) changes significantly with the population age, going from 0 for very young stars to a maximum at about $0.5 \mathrm{Gyr}$, then falling to 0 again for stars older than $5 \mathrm{Gyr}$. This behaviour agrees with observational data for AGB stars in LMC star clusters (see e.g. Frogel et al. 1990).
Changing now from a constant to a variable SFR, the age distribution can be constructed from that in Fig. 7 by giving relative weights to age intervals that have different $\mathrm{C} / \mathrm{M}$ ratios. This makes it clear that the $\mathrm{C} / \mathrm{M}$ ratio must depend on the shape of the SFR function. It is also evident, from the figure, that by giving more weight to stars recently formed (decreasing SFR), we get higher $\mathrm{C} / \mathrm{M}$ ratios (and vice-versa for increasing SFR).

Thus, the $\mathrm{C} / \mathrm{M}$ ratio of a stellar population depends both on the metallicity and on the SFR. At lower metallicities the $\mathrm{C} / \mathrm{M}$ ratio is on average higher than at higher metallicities. Moreover variations of the SFR cause variations of the $\mathrm{C} / \mathrm{M}$ ratio that are slightly higher at higher metallicities than at lower metallicities.

\subsubsection{A more realistic LMC model}

So far, we have used the quite unrealistic cases of constant AMR, i.e. no chemical evolution over the galaxy history, together with very simplified prescriptions for the SFR. We will now describe a simulation that adopts a SFR and an AMR derived from observations (Fig. 8). The SFR is from the analysis, via an objective method, of optical HST photometry reaching the oldest main sequence turn-off in a bar field (Holtzman et al. 1999). The AMR is from chemical evolution models (Pagel \& Tautvaisiene 1999), but is shown to agree very well with independent determinations of chemical abundances and ages of star clusters in the LMC.

The resulting CMD (Fig. 8) represent the observations rather well, and should be compared to actual near-IR data, for instance the figures in van der Marel \& Cioni (2000c) and Marigo et al. (2003), the O-rich TP-AGB stars form two distinct almost-vertical sequences in the CMD: the first to the blue (left) and the second to the red (right). The first extends to bright magnitudes; the second sequence is right above the RGB. It is somewhat limited in magnitude (the exact extension depending mostly on the assumed metallicity); both sequences merge at lower luminosities, at about $K \sim 11.6$ for $Z=0.008$. In Fig. 6 , it is clear that the bluest sequence is caused by the younger populations (age less than a few Myr), whereas 


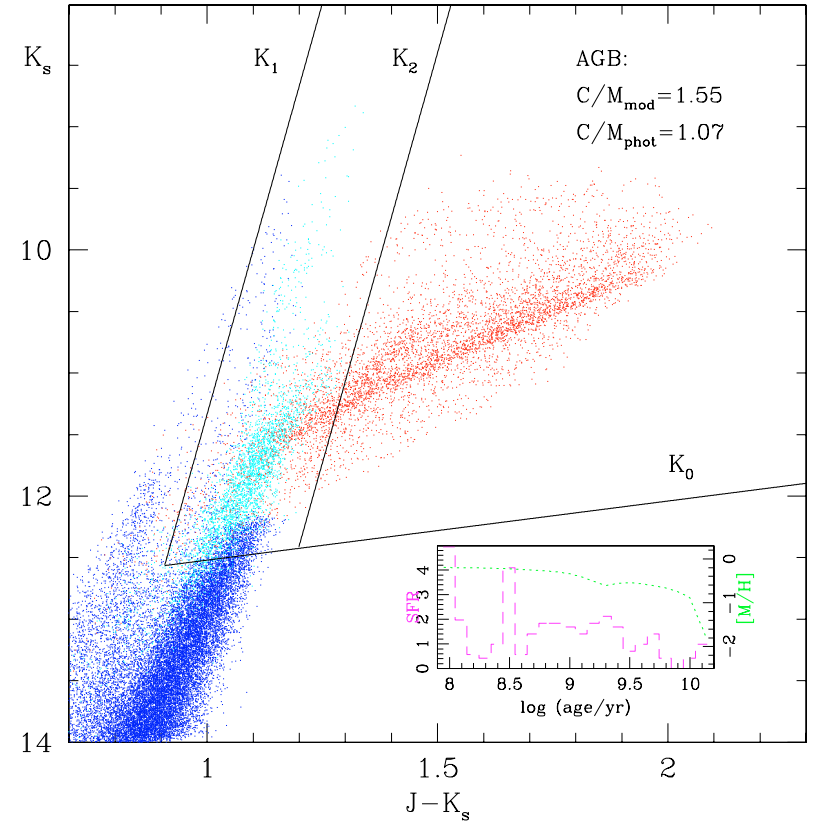

Fig. 8. Simulated CMD for a model that assumes observed SFR and AMR for a LMC bar field using Holtzman et al.'s (1999) SFR. The AMR of Pagel \& Tautvaisiene (1999) was used. In this simulation the O-rich TP-AGB stars make a single and straight sequence in the CMD, being much better defined than in previous constant-metallicity simulations of Figs. 4 and 6.

the reddest is caused by a population older than a few Gyr. The two features are separate in the CMD because the stars of intermediate age expected in between the two sequences have become C-type TP-AGB stars and they now form the red tail thus leaving an empty "valley" in the RG- and the AG-Branch.

In the more realistic simulation of Fig. 8, these two sequences of O-rich TP-AGB stars actually merge into a single sequence. This happens because the metallicity difference between young and old populations has now been taken into account: the young metal-rich and old metal-poor populations with age and metallicity as defined by the underlying AMR now happen to have about the same colours. And in fact, Magellanic Cloud observations (DENIS, 2MASS) present a single sequence of M-type AGB stars on top of the RGB, exactly as in our simulation of Fig. 8.

\section{Comparison between observed and theoretical distribution}

We have defined clear criteria for extracting C- and M-type TP-AGB stars from both data and theoretical simulations. Let us now compare the model and data for the LMC in more detail. First of all we compare the observed and theoretical LFs for an inner section. We have chosen the ring 0 and sector E-NE (cf. Sect. 2.4) because for this region the number of stars is large and crowding is not a problem. The left panel of Fig. 9 shows the LF results compared to our "realistic" LMC model discussed in Sect. 3.3.3 using the SFR and AMR as published.
The number of simulated stars has been normalized to give the same number of C-type stars as in the observations.

In Fig. 9 (left) the photometric criteria for selecting $\mathrm{C}$ and M AGB stars, shown in the bottom panel, provide LFs in good agreement with the model distribution in the upper panel. Differences between the two criteria appear only at the faintest magnitude bins, as expected. Looking at the bottom panels, we can notice the good description of the LF shapes for both $M$ and C-type stars. But the number of M-type AGB stars predicted by the model is just about half of the observed ones. Thus, this particular model strongly overpredicts the $\mathrm{C} / \mathrm{M}$ ratio of AGB stars.

We simulated the Galaxy foreground with the TRILEGAL code - as described in Girardi et al. (2004) and verified that the contamination of the CMD in the M-star region by foreground objects is very modest, and has no effect on the observed $\mathrm{C} / \mathrm{M}$ ratio. Thus, we conclude that our "realistic" LMC model has a problem. One possibility is that the TP-AGB models by Marigo et al. in some way overestimate the number of $\mathrm{C}$ stars; however this seems quite unlikely since their lifetimes in the $\mathrm{C}$-star phase agree with those derived from C-star counts in LMC clusters (Girardi \& Marigo 2002). Alternatively, the fraction of M-type AGB stars may be underestimated, a possibility that is presently under investigation. Another possibility is that the SFR we use - as derived from other authors using completely independent data, and regarding different regions of the $\mathrm{LMC}$ - is not appropriate for the region we are looking at.

Since we cannot, at this stage, clarify the origin of the problem, we proceed in the following way: we fix the AMR of the LMC, and change the family of SFR given by Eq. (8). The parameter $\alpha$ is let vary and then for each simulation we compare the derived and observed LF, separately for $\mathrm{C}$ and $\mathrm{M}$ stars. The comparison is based on a $\chi^{2}$ test. For example in Fig. $9 \chi_{\mathrm{C}}^{2}$ and $\chi_{\mathrm{M}}^{2}$ indicate the probability that the observed distribution is represented by the theoretical distribution (or the probability of getting the $\chi^{2}$ value obtained comparing both distributions). It is clear that the particular model shown in the left panel of Fig. 9 represents rather well the distribution of $\mathrm{C}$ stars at the $96.1 \%$ level while the distribution of $\mathrm{M}$ stars is far from the one predicted. For comparison, in the right panel we plot the results for the SFR that best fits the LFs for both C-and M-type stars ( $\alpha=1000)$; in this case, the model well represents the distribution of $\mathrm{C}$ stars at the $94.5 \%$ level and the one of M stars at the $58.6 \%$ level.

For each sector in each ring we have calculated the probability as above for each combination of SFR and metallicity $Z$. The $Z$ that gives the best fit is then interpreted as the best model for the stellar population in that particular sector. We adopt a constant AMR, i.e. the same $Z$ for all ages, which is an acceptable approximation since the metallicities that count most in defining the model LFs are those at young ages (say less than 3 Gyr) for which little galactic chemical evolution is expected. Note also that we select $\mathrm{C}$ and $\mathrm{M}$ stars with the help of lines in the diagrams, lines that depend on the chosen metallicity $Z$.

Since our best fitting model results from a series of approximations - both in the TP-AGB model, and in the assumption of 

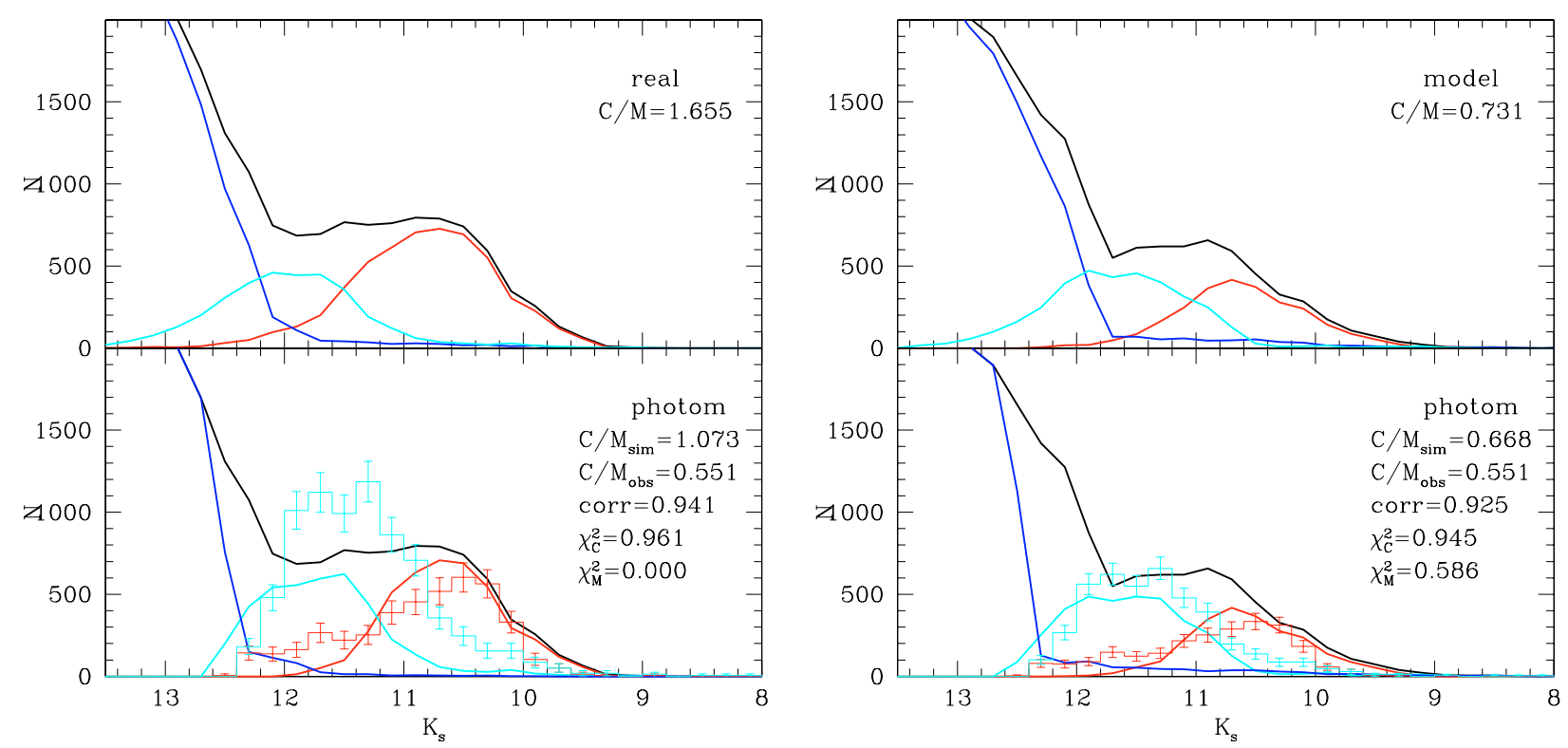

Fig. 9. LFs for a realistic LMC model (left) and for a model that fits rather well both the distribution of C and M stars (right) of sector E-NE of ring 0 . The continuous lines show model results: cyan $=$ O-rich TP-AGB, red $=$ C-rich TP-AGB, blue $=$ other stars, black $=$ total. Upper panel: using the modelling criteria to classify TP-AGB stars; bottom panel: using the photometric criteria. In the latter case, LFs are compared to LMC data (histograms). The number counts in the simulations have been normalized to provide the same C-star number as in the data. In each panel of the bottom row the labels inside each figure give the observed and photometric $\mathrm{C} / \mathrm{M}$ ratios as well as the correlation coefficient between the theoretical and observed distribution of $\mathrm{C}$ stars. The last two numbers indicate the probability that the observed distribution is represented by the given model distribution; this is the same probability of obtaining the $\chi^{2}$ derived from the comparison between the two distributions.

exponential SFRs - the best fit we get for a single LMC region may not be the best possible solution. Therefore, we should not overinterpret the results in terms of the derived metallicities and mean ages. Much more important than the absolute values of these parameter, are their variations across the LMC. Our next goal is to map these variations.

In Fig. 10 maps of metallicity across the LMC area are shown separately for $\mathrm{C}$ and $\mathrm{M}$ stars for each SFR. Contour values directly correspond to metallicity expressed as values of $Z$. The distribution of the maximum probability is also shown and indicates how significant a metallicity map is.

In order to build these maps, because we dispose of numbers (probability, metallicity, SFR) for a given sector (this is necessary in order to obtain a statistical significant LF), we first created a grid of 25921 points in the plane $(-8: 8,-8: 8)$ equally spaced with a step of 0.1 . Second we assigned to each point the quantity in which we are interested (for example the most probable metallicity) accordingly to which sector a point belongs. Third we re-binned the distribution of values in bins equal to 0.4 (this corresponds to a resolution of $0.16 \mathrm{deg}^{2}$ ) and applied a boxcar smoothing of width $=2$. Finally, we constructed greyscale maps where darker regions correspond to higher numbers.

\subsection{The most probable metallicity and SFR distributions}

Combining the information in each of the maps in Fig. 10 we have constructed maps that for $\mathrm{C}$ and $\mathrm{M}$ stars separately show the best combination of metallicity and SFR. In practice we
Table 1. Mean age of all stars formed accordingly to a specific rate of star formation.

\begin{tabular}{cc}
\hline \hline$\alpha$ & $\langle$ Age $($ Gyr $)\rangle$ \\
\hline-2 & 2.0 \\
-5 & 3.9 \\
$+\infty$ & 6.3 \\
5 & 8.7 \\
2 & 10.6 \\
\hline
\end{tabular}

show the metallicity that corresponds to the maximum probability among the different SFRs (i.e. for the C stars in the top row of Fig. 10 the most probable value in each bin). The result is shown in Fig. 11 where the map of best metallicity, SFR and corresponding probability are shown, again separately for C (top row) and M stars (bottom row). Note that to each SFR we have assigned the mean age of all stars that formed accordingly to that particular SFR because this quantity, contrary to the mean age of AGB stars only, does not depend on metallicity (Table 1). Therefore contour values correspond to the mean age of the local population.

Figure 12 shows the distribution of the number of $\mathrm{C}$ and $\mathrm{M}$ stars and of their $\mathrm{C} / \mathrm{M}$ ratio across the LMC with the same binning as in the other maps. A high resolution version of the distribution of the $\mathrm{C} / \mathrm{M}$ ratio as obtained in Paper $\mathrm{I}$ is also shown. The effect of binning on smoothing galaxy features is clear, but major trends are conserved. For example a comparison of both $\mathrm{C} / \mathrm{M}$ ratio distributions shows a dark region (i.e. a region with a high $\mathrm{C} / \mathrm{M}$ ratio) to the $\mathrm{S}$ of the 

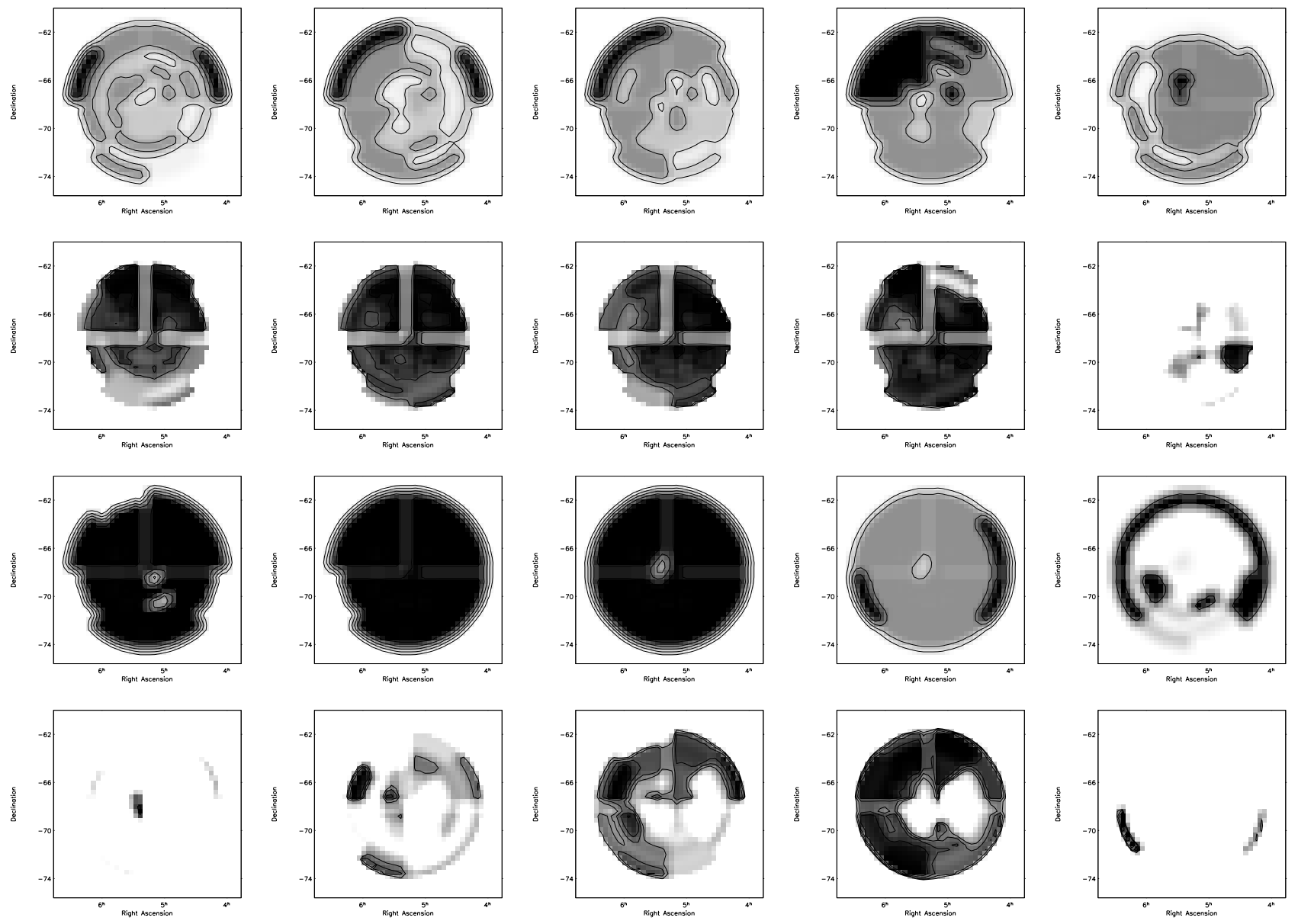

Fig. 10. Distribution of the metallicity that corresponds to the maximum probability for a given SFR for C stars (first row) and M stars (third row). From left to right the SFR corresponds to a population with a mean age of 2, 3.9, 6.3, 8.7 and 10.6 Gyr. Metallicity contours are at $Z=0.003-0.015$ with a step of 0.003 . Darker regions correspond to higher values. The probability distributions associated with each SFR are shown in the second row for C stars (the grey scale shows only values above 0.8 and contours are at $0.93,0.96$, and 0.99 ) and in the fourth row for $\mathrm{M}$ stars (the grey scale shows only values above 0.6 and contours are at $0.8,0.85,0.9,0.95$ ).

LMC extending to the NW, another approximately circular region is present NW of the galaxy centre. The first dark patch is recognizable also in the high-resolution map as well as the patch close to the centre. The latter is emphasized in the lowresolution map because of the larger bin size although it remains surrounded by a region of much lower $\mathrm{C} / \mathrm{M}$ ratio values. High resolution maps of all AGB stars (C plus $\mathrm{M}$ stars) can be found in Cioni et al. (2000b).

The distribution of $\mathrm{C}$ stars is overall best described by $Z=$ 0.006 except in the NE region (towards the Milky Way plane) as well as in two approximately circular inner regions where the metallicity appears to be higher, and in the SW, towards the SMC, encompassing the bar region where the metallicity is perhaps lower. The stellar population has a rather patchy distribution of age: it is on average $8 \mathrm{Gyr}$ old in the S and NE with inner areas with stars at least $3 \mathrm{Gyr}$ younger. The probability distribution obtained from fitting the LF of C-rich AGB stars is overall above $80 \%$ with most values above $97 \%$; this provides an excellent constraint to the distribution of metallicity and look-back-time. Note that the bar of the galaxy does not emerge from these maps, perhaps indicating that its formation was not coeval.
Similar maps for $\mathrm{M}$ stars indicate an overall metallicity, in agreement with that derived from $C$ stars, of $Z=0.006$ except along the bar of the galaxy and continuing to the outer $\mathrm{W}$ region, where the metallicity could be higher. The distribution of the mean age of the stellar population is rather uniform and indicates a mean age of 8 Gyr except for three well defined regions: an older population to the $\mathrm{E}$ and a younger population close to the center and; on the $\mathrm{W}$ end of the bar region. However the probability distribution provides a weaker constraint especially in this region.

\subsection{Projection effect}

The orientation of the LMC disk has been determined by van der Marel \& Cioni (2001) analysing the brightness variation of features in the colour-magnitude diagram as a function of position within the galaxy. In particular the mode of the distribution of AGB stars, independently of their spectral type, of C-rich stars only and of the tip of the RGB. All these tracers produced a sinusoidal variation with an amplitude of about 0.25 mag as a function of angular position in the galaxy. 

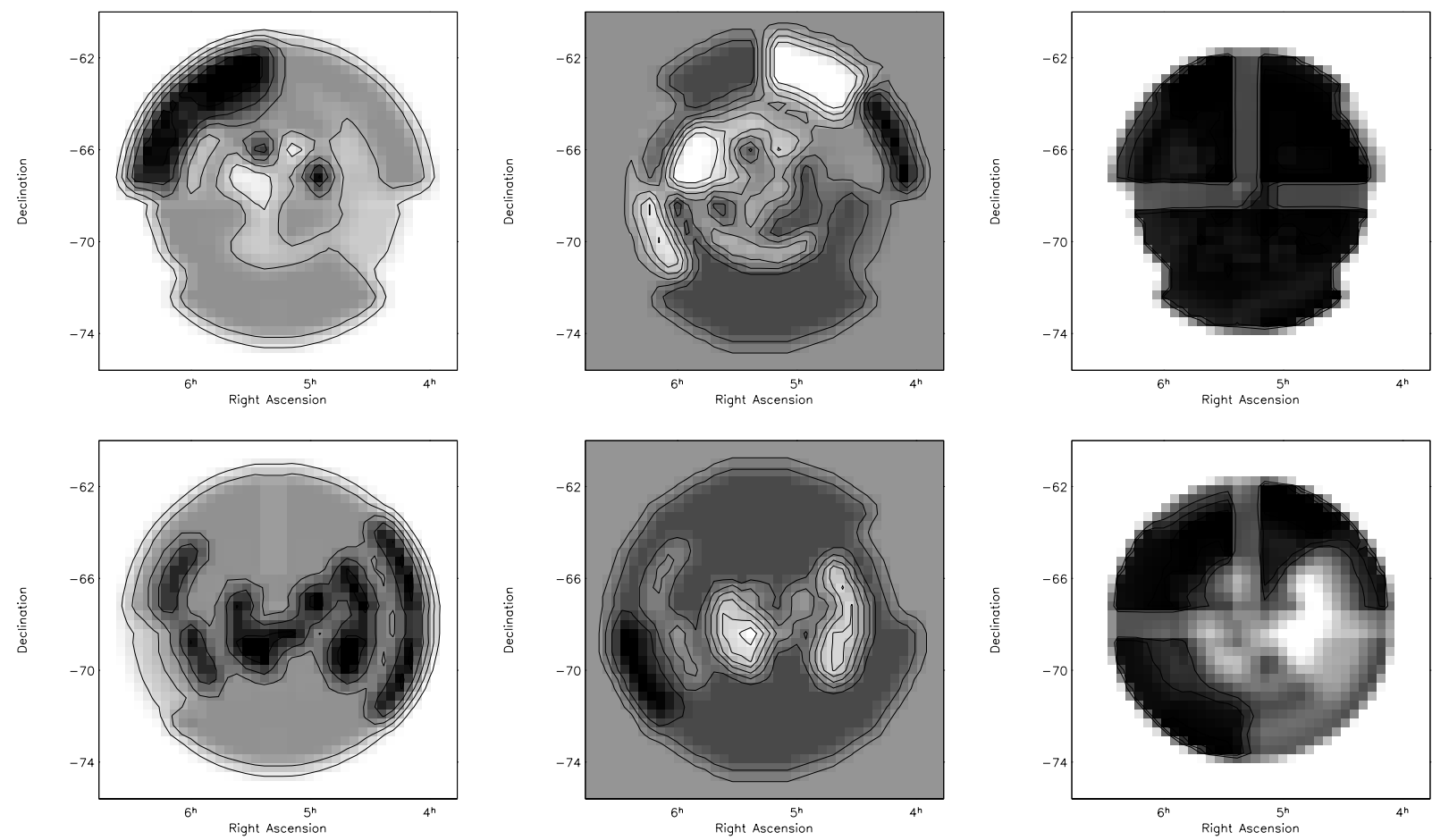

Fig. 11. Top row: distribution of the most probable metallicity (left - contours are at $A=0.003-0.015$ with a step of 0.003 ), SFR (middle contours are from 3 to $9 \mathrm{Gyr}$ with a step of 1) and the corresponding probability (right - the grey scale shows values above 0.8 and contours are at $0.97,0.98$ and 0.99 ) for C stars. Bottom row: the same distributions, with the same contour values, for M stars. Darker regions correspond to higher numbers.
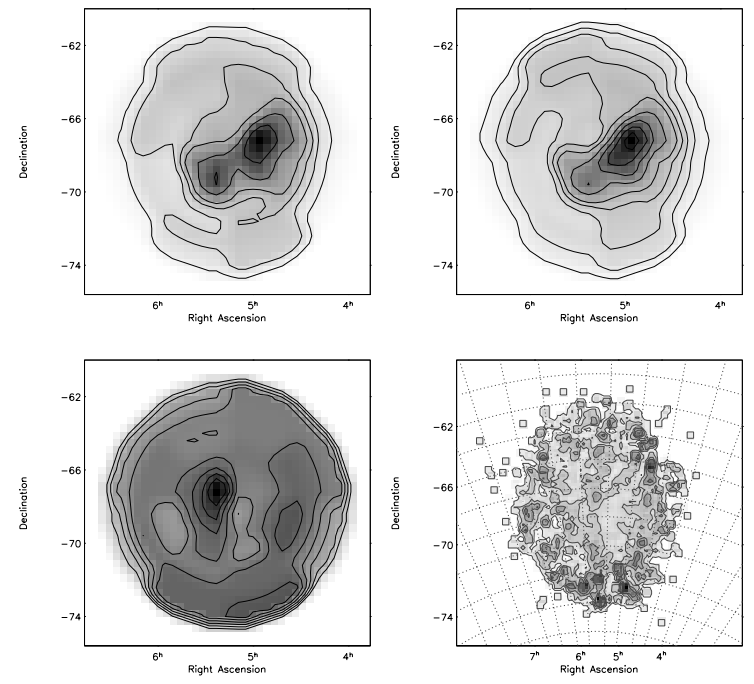

Fig. 12. Top row: distribution of the number of $\mathrm{C}$ (contours are at 50 , 100,150 and then at 200-500 with a step of 100) and M (contours are at $100-500$ with a step of 100 and then at $700-1300$ with a step of 200) stars. Bottom row: distribution of the $\mathrm{C} / \mathrm{M}$ ratio binned as in this work $\left(0.16 \mathrm{deg}^{2} / \mathrm{bin}\right.$ - contours are at $0.2-0.55$ with a step of 0.05$)$ and as in Paper I ( $0.04 \mathrm{deg}^{2} / \mathrm{bin}$ - zoom into original figure). Note that here because we need a significant number of stars to fit the LF we do not reach a resolution as high as in Paper I while maintaining the same global features (see text).

A best fit geometrical model was used to infer a value for the inclination and position angle of the line of nodes.
Table 2. Magnitude shift due to the LMC orientation. The shift is the same for rings number 2, 3, 4 and 5 .

\begin{tabular}{ll|ll}
\hline \hline Sector & Shift & Sector & Shift \\
\hline E-NE & -0.12 & W-SW & 0.12 \\
NE-N & -0.10 & SW-S & 0.10 \\
N-NW & -0.02 & S-SE & 0.02 \\
NW-W & 0.07 & SE-E & -0.09 \\
\hline
\end{tabular}

The detected amplitude is of the same order of size of the bins in the maps presented above, therefore it may affect the shape of the distribution of either C-rich or O-rich AGB stars. For example a shift of $0.1 \mathrm{mag}$ implies that half of the sources in a given bin will move to the adjacent bin. In order to investigate the strength of this effect we have applied a correction to each sectors within rings 2 and 5 following the best fit model used by van der Marel \& Cioni (2001). The correction is the same for each ring and is listed in Table 2. Although van der Marel \& Cioni (2001) also detected a radial variation (i.e. as a function of ring) it is not very well constrained. Note also that no correction was applied to the inner most rings (0 and 1). This region, strongly influenced by the effect of the bar, requires further study.

Figure 13 shows as an example the distribution of C-rich and O-rich AGB stars with and without correction. In this case (sector E-NE of ring 2) a shift of -0.12 mag is applied and the 


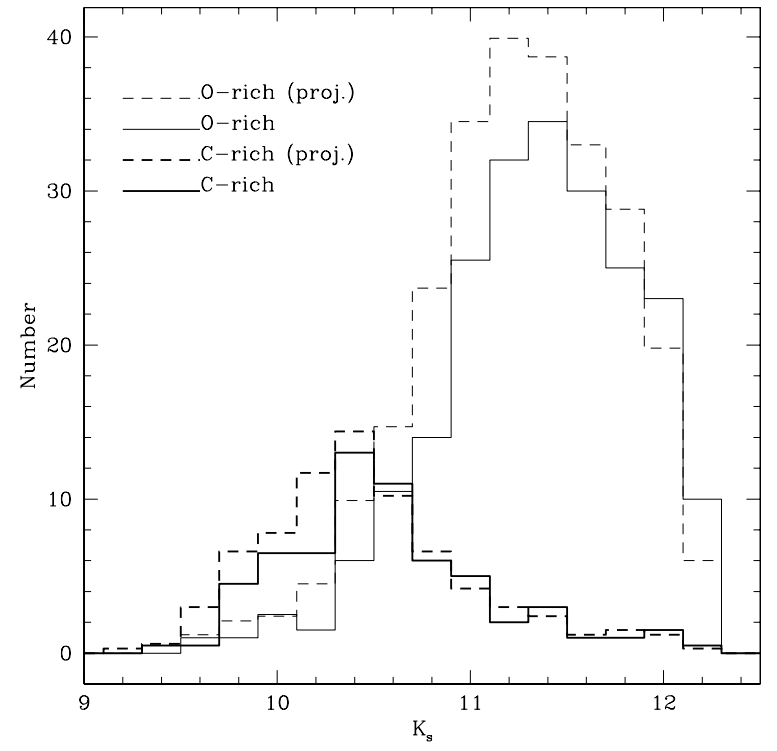

Fig. 13. Magnitude distribution of C-rich and O-rich AGB stars in sector E-NE of ring 2 with (dashed lines) and without (continuous line) correcting for the orientantion of the LMC.

histogram moves to brighter magnitudes as well as changing in shape. Note the distribution of C-rich stars around $K_{\mathrm{s}}=10.2$.

Figure 14 shows the same maps as in Fig. 11 but after correcting for the orientation of the LMC. The fit of the observed distribution of both $\mathrm{C}$ and $\mathrm{M}$ stars occur at almost equal probabilities while there are considerable variations in the distribution of both metallicity and mean-age across the area of the galaxy. In particular the outermost metal rich E-NE region suggested by $\mathrm{C}$ stars has moved slightly $\mathrm{S}$. The metallicity remains low towards the SMC. The distribution of the meanage of the stellar population, although still appearing rather patchy, indicates an inner ring/region more metal rich than the outer ring/region. M stars support the same overall metallicity $Z=0.006$ but the region of high metallicity that previously coincided with the bar of the galaxy is now stretching from $E$ to SW encompassing the $\mathrm{E}$ and central bar region but not the $\mathrm{W}$ end of it. The same structure corresponds to a younger population (about $3 \mathrm{Gyr}$ on average) compared to the population of the overall disk, although in the SE a region with an older population is still present.

\section{Discussion}

\subsection{How well does the $\mathrm{C} / \mathrm{M}$ ratio alone trace metallicity?}

In Paper I the distribution of the $\mathrm{C} / \mathrm{M}$ ratio has been interpreted purely as a tracer of metallicity concluding that the LMC shows the typical metallicity pattern, as in other galaxies, of decreasing metallicity towards its outskirts. In this paper we have shown that the $\mathrm{C} / \mathrm{M}$ ratio depends also on the SFR and we have created maps of metallicity, separately for $\mathrm{C}$ and $\mathrm{M}$ stars, that account for this effect. After this analysis are the conclusions of Paper I still valid?
Magnitude variations of the order of those applied in the previous section have a minor effect on the distribution of the $\mathrm{C} / \mathrm{M}$ ratio. At the brightest magnitudes there are overall very few sources while at the faintest magnitudes the contamination with RGB stars and the larger photometric errors involved play a larger role in assigning a source to a given bin than a shift due to the viewing angles of the galaxy. Only in regions with overall low number statistics is the $\mathrm{C} / \mathrm{M}$ ratio influenced by the orientation of the galaxy. A precise correction would also include a radial component that in the case of van der Marel \& Cioni (2001) was poorly constrained although present. This means that only the tangential component of the orientation has been corrected for. Considering also that for the SMC (Paper III) a correction is not known at present, and we would like to analyse both galaxies consistently, in the following discussion we compare the distribution of the $\mathrm{C} / \mathrm{M}$ ratio (Fig. 12) with the maps presented in Fig. 11.

The distribution of the C/M ratio in the LMC (Fig. 12) shows a higher ratio to the $\mathrm{S}$. This indeed corresponds to regions of low metallicity in both the metallicity map obtained from $\mathrm{C}$ and $\mathrm{M}$ stars. We can safely conclude that in this region the $\mathrm{C} / \mathrm{M}$ ratio alone is a good tracer of metallicity. Extending to the SW high $\mathrm{C} / \mathrm{M}$ values indicate a low metallicity in agreement with the metallicity derived from C-stars but O-rich stars support a higher metallicity in this region, although the probability is low $(<60 \%)$. On the periphery of the LMC, still in the $\mathrm{SW}$, the $\mathrm{C} / \mathrm{M}$ ratio decreases supporting a higher metallicity in agreement with that traced by $\mathrm{M}$ stars. In this region there are not enough $\mathrm{C}$ stars to produce a reasonable fit of their luminosity function, the same is true in the SE. Note that the distribution of the number of $\mathrm{C}$ and $\mathrm{M}$ stars shows that $\mathrm{M}$ stars extend in number much further out compared to $\mathrm{C}$ stars. The well defined region of high metallicity (NE) obtained from $\mathrm{C}$ stars is also high in metals from $\mathrm{M}$ stars although the latter spreads across a much larger area. In this region the $\mathrm{C} / \mathrm{M}$ ratio has intermediate values but clearly decreases to the periphery it is thus plausible to conclude that towards the NE of the LMC the metallicity is high. In the central part of the galaxy the C/M ratio indicates a region of low metallicity. The $\mathrm{C}$ star metallicity distribution supports this finding although there is a dichotomy in the sense that the northern most part of this small region could be metal rich while the southernmost part of it metal poor. Even though the binning plays a major role in exactly constraining the location of this inner region, it may correspond to Shapley constellation III where star formation is currently taking place. This explains the young population inferred from $\mathrm{M}$ stars as well as an underlying older population inferred from $\mathrm{C}$ stars. Therefore in this region the $\mathrm{C} / \mathrm{M}$ ratio is strongly influenced by age. The $\mathrm{C} / \mathrm{M}$ ratio is not a good tracer of metallicity if the chemical composition of a system varies on time scales shorter than about 1 Gyr (Mouhcine \& Lançon 2002).

Although at first sight the metallicity maps obtained from $\mathrm{C}$ and $\mathrm{M}$ stars and the distribution of the $\mathrm{C} / \mathrm{M}$ ratio do not look much alike they have similar trends. This is the strength of the $\mathrm{C} / \mathrm{M}$ ratio alone while for a more accurate evaluation of metallicity a knowledge of the age of the population is essential. A comparison between the $\mathrm{C} / \mathrm{M}$ ratio (Fig. 12) and the metallicity distributions shown in Fig. 14 allows us to reach similar 

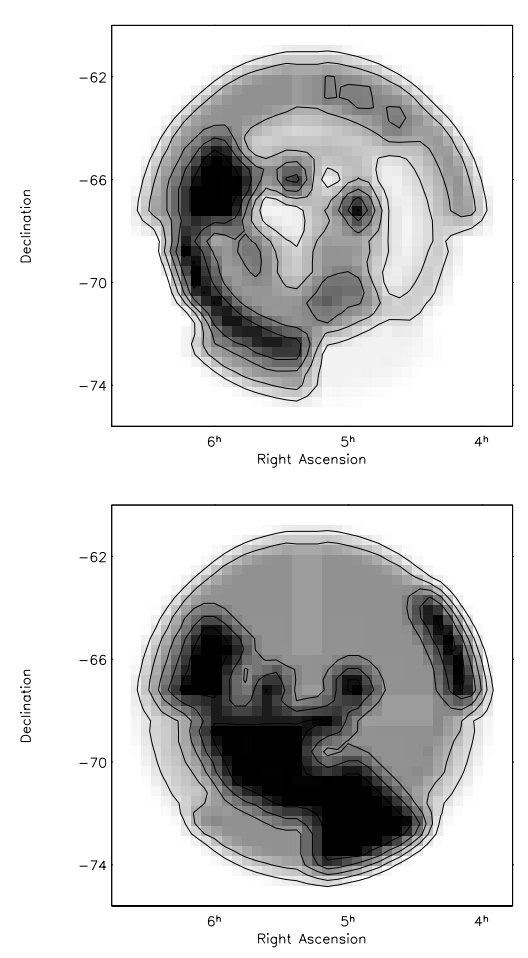
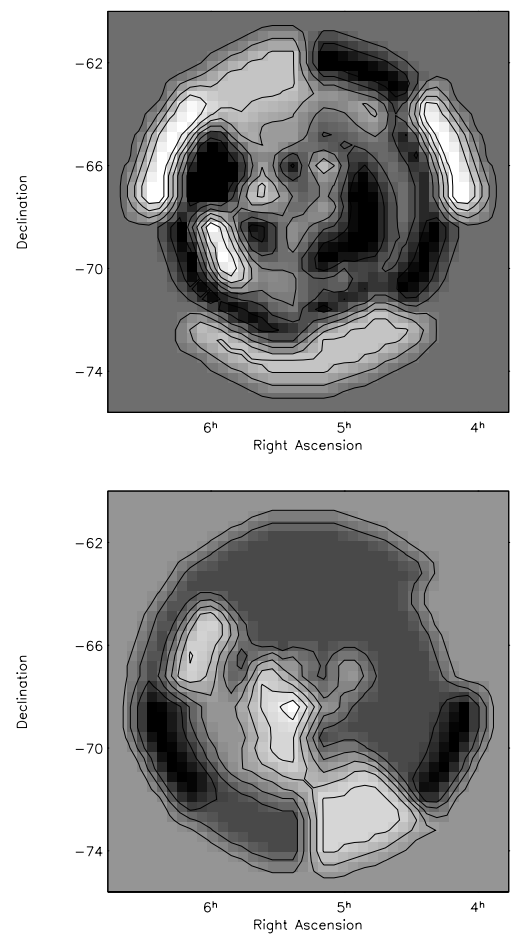
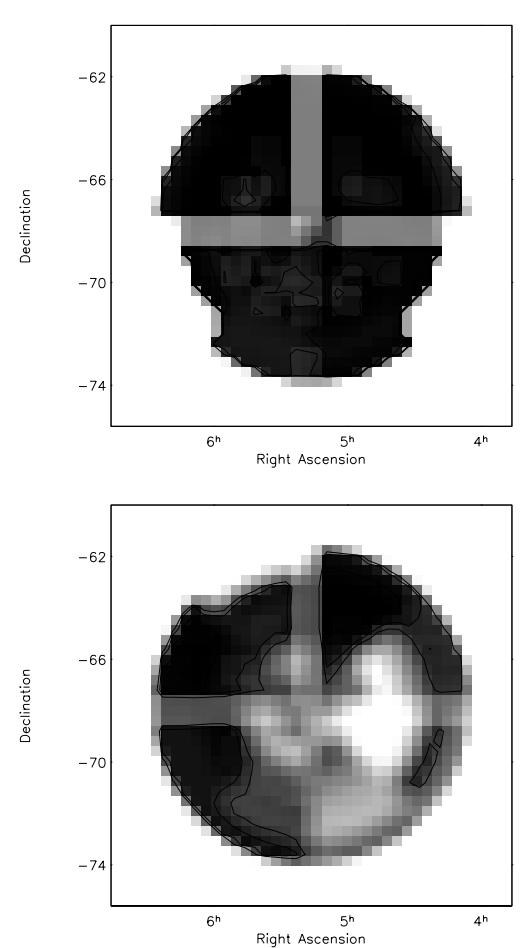

Fig. 14. The same as Fig. 11 but after correcting each sector in rings number $2-5$ for the LMC orientation as in Table 2. Contour values are the same as in Fig. 11.

conclusions in those regions where the probability (i.e. the level of significance) is high, in particular for $\mathrm{C}$ stars.

Finally the spread in metallicity within both Magellanic Clouds derived in Paper I amounts to 0.75 dex, this is in very good agreement with 0.7 dex derived from $\mathrm{C}$ stars and $\mathrm{M}$ stars (i.e. the difference between the low and high contour converted into $[\mathrm{M} / \mathrm{H}]$ assuming $\left.Z_{\odot}=0.019\right)$.

\subsection{The mean age distribution}

Interpreting the magnitude distribution of $\mathrm{C}$ and $\mathrm{M}$ stars we derive that most of the LMC population is on average about 5-6 Gyr old (Fig. 11), supporting a population that is predominatly of intermediate age. There are, however, well defined regions with older or younger stars especially in the northen hemisphere of the galaxy compared to the southerm hemisphere that appears more homogeneous, unless a correction for the LMC viewing angles is applied. There is a clear indication that the bar of the galaxy contains a composite stellar population (i.e. it did not form at the same time). $\mathrm{C}$ stars indicate that the periphery of the galaxy is not systematically older than the inner region, contrary to $\mathrm{M}$ stars, although the indication provided by $\mathrm{M}$ stars is less significant (i.e. they correspond to a lower $\chi^{2}$ value). If the distribution of stars is corrected for the projection of the galaxy there appear to be younger $\mathrm{C}$ stars in its periphery. Note that this is further out than the observations by Costa \& Frogel (1996) who claimed a similar result.

\section{Summary and conclusions}

In this paper we compare the observed $K_{\mathrm{s}}$ magnitude distribution of $\mathrm{C}$ and $\mathrm{M}$ stars within sectors entirely covering the LMC, of a suitable size to provide a statistically significant sample, with theoretical distributions. These have been obtained using the population synthesis code TRILEGAL (Girardi et al. 2005), employing suitable stellar evolutionary tracks for stars of different mass and in particular using Marigo et al. (2003) models for thermally pulsing AGB stars. This allows us to well describe the behaviour of both $\mathrm{C}$ and $\mathrm{M}$ type stars as a function of $K_{\mathrm{s}}$ magnitude. The quality of the comparison has been quantified using the $\chi^{2}$ test.

Surface maps indicating the most probable metallicity and mean age distribution have been obtained varying one or both parameters at the same time. Results have been compared with the information available from previous studies, although they covered only a limited area of the LMC. With respect to the conclusions reached in Paper I, the $\mathrm{C} / \mathrm{M}$ ratio is influenced by the age of the population where it is calculated, however it remains a good tracer of metallicity only if the population is on average older than a few Gyr. Note that here we refer to the mean age of the stellar population in a given spatial location and not to the precise age at which that particular population began forming stars. According to the LMC structure parameters derived by van der Marel \& Cioni (2001) we have created new surface maps that account for the viewing angles of the LMC. Non-negligible differences were obtained in the distribution of both metallicity and mean age of the underlying stellar population.

Our best fit models indicate that stars in the LMC are on average 5-6 Gyr old, and that the metallicity of most stars is consistent with $Z=0.006$. However, since TP-AGB models are not perfect, and since we are testing just a family (the exponential ones) among many possible SFR histories, these average 
values of age and metallicity may be affected by systematic errors, and are likely to change as we adopt improved TPAGB models, and test other possible SFR functions. Instead, we consider to be more significant the detected variations of these mean age and metallicity values across the LMC disk. In fact, we find distinct regions in the LMC with an older or a younger mean age (i.e. Shapley III). There is also a clear indication that the metallicity is higher towards the plane of the Milky Way and lower towards the SMC. This perhaps traces the dynamical history of the galaxy (i.e. the interaction with its companions) for which a final understanding will be provided from a detailed kinematic study of its stellar population.

Acknowledgements. L.G. is indebted to M. Salaris and M. Groenewegen for the frequent sharing of information on several aspects of simulating CMDs for Local Group galaxies.

\section{References}

Alcock, C., Allsman, R. A., Alves, D. R., et al. 1999, AJ, 117, 920 Ardeberg, A. 1976, A\&A, 46, 87

Ardeberg, A., Gustafsson, B., Linde, P., \& Nissen, P.-E. 1997, A\&A, 322, L13

Bekki, K., Couch, W. J., Beasley, M. A., et al. 2004, ApJ, 610, L93

Bergeat, J., Knapik, A., \& Rutily, B. 2001, A\&A, 369, 178

Bertelli, G., Mateo, M., Chiosi, C., \& Bressan, A. 1992, ApJ, 388, 400

Bertelli, G., Bressan, A., Chiosi, C., et al. 1994, A\&AS, 106, 275

Blanco, V. M., McCarthy, M. F., \& Blanco, B. M. 1980, ApJ, 242, 938

Bonatto, C., Bica, E., \& Santos, J. F. C. 2005, A\&A, 433, 917

Butcher, H. 1977, ApJ, 216, 372

Castelli, F., Gratton, R. G., \& Kurucz, R. L. 1997, A\&A, 324, 432

Chabrier, G. 2001, ApJ, 554, 1274

Cioni, M.-R. L., \& Habing, H. J. 2003, A\&A, 402, 133 (Paper I)

Cioni, M.-R. L., Loup, C., Habing, H. J., et al. 2000a, A\&AS, 144, 235

Cioni, M.-R. L., Habing, H. J., \& Israel, F. P. 2000b, A\&A, 358, L9

Cioni, M.-R. L., van der Marel, R. P., Loup, C., \& Habing, H. J. 2000c, A\&A, 359, 601

Cioni, M.-R. L., Marquette, J. B., Loup, C., et al. 2001, A\&A, 377, 945

Cioni, M.-R. L., Blommaert, J. A. D. L., Groenewegen, M. A. T., et al. 2003, A\&A, 406, 51

Costa, E., \& Frogel, J. A. 1996, AJ, 112, 2607

Delmotte, N., Loup, C., Egret, D., et al. 2002, A\&A, 396, 143

Elson, R. A. W., Gilmore, G. F., \& Santiago, B. X. 1997, MNRAS, 289,157

Feitzinger, V., Glassgold, A. E., Gerola, H., \& Sneiden, P. E. 1981, A\&A, 98, 371

Fluks, M. A., Plez, B., Thé, P. S., et al. 1994, A\&AS, 141, 313

Frogel, J. A., \& Blanco, V. M. 1983, ApJ, 274, L57

Frogel, J. A., Mould, J., \& Blanco, V. M. 1990, ApJ, 352, 96

Gallagher, J. S., Mould, J., de Feijter, E., et al. 1996, ApJ, 466, 732

Gallart, C., Stetson, E., Hardy, E., et al. 2004, ApJ, 614, L109

Geha, M. C., Holtzman, J. A., Mould, J. R., et al. 1998, AJ, 115, 1045

Girardi, L., Bressan, A., Bertelli, G., \& Chiosi, C. 2000, A\&AS, 141, 371
Girardi, L., Bertelli, G., Bressan, A., et al. 2002, A\&A, 391, 195

Girardi, L., Groenewegen, M. A. T., Hatziminaoglou, E., \& da Costa, L. 2005, A\&A, 436, 895

Glass, I. S. 1999, in The Handbook of Infrared Astronomy (Cambridge University Press)

Glass, I. S., Schultheis, M., \& Cioni, M.-R. L. 2004, A\&A, 427, 945

Groenewegen, M. A. T. 2004, A\&A, 425, 595

Hardy, E., Buonanno, R., Corsi, C. E., et al. 1984, ApJ, 278, 592

Hodge, P. W. 1973, AJ, 78, 807

Hodge, P. W. 1988, PASP, 100, 576

Holtzman, J. A., Mould, J. R., Gallagher, J. S., et al. 1997, AJ, 113, 656

Holtzman, J. A., Gallagher, J. S., Cole, A. A., et al. 1999, AJ, 188, 2262

Isserstedt, J. 1984, A\&A, 131, 347

Izzard, R. G., Tout, C. A., Karakas, A. I., \& Pols, O. R. 2004, MNRAS, 350, 407

Javiel, S. C., Santiago, B. X., \& Kerber, L. O. 2005, A\&A, 431, 73

Kontizas, M., Margoudaki, F., Kontizas, E., et al. 2004, BaltA, 13, 632

Kurucz, R. L. 1992, IAUS, 149, 225

Lah, P., Kiss, L. L., \& Bedding, T. R. 2005, MNRAS, 359, L42

Marigo, P. 2001, A\&A, 370, 194

Marigo, P. 2002, A\&A, 387, 507

Marigo, P., Girardi, L., \& Bressan, A. 1999, A\&A, 344, 123

Marigo, P., Girardi, L., \& Chiosi, C. 2003, A\&A, 403, 225

Mouhcine, M., \& Lançon, A. 2002, A\&A, 393, 149

Olsen, K. A. G. 1999, AJ, 117, 2244

Olson, G. L., \& Pefia, J. H. 1976, ApJ, 205, 527

Ostlie, D. A., \& Cox, A. N. 1986, ApJ, 311, 864

Pagel, B. E. J., \& Tautvaisiene, G. 1999, Ap\&SS, 265, 461 358, 1215

Reid, N., \& Mould, J. 984, ApJ, 284, 98

Rocca-Volmerange, B., Lequeux, J., \& Maucherat-Joubert, M. 1981, A\&A, 104, 177

Skrutskie, M. 1998, in The Impact of Near-Infrared Sky Surveys on Galactic and Extragalactic Astronomy, Proc. of the 3rd Euroconference on Near-Infrared Surveys, ed. N. Epchtein, Astrophysics and Space Science library, 230, 11 (Dordrecht: Kluwer)

Smecker-Hane, T. A., Cole, A. A., \& Gallagher, J. S. 1999, ApJ, 566, 239

Stappers, B. W., Mould, J. R., Sebo, K. M., et al. 1997, PASP, 109, 292

Stryker, L. L. 1984, ApJS, 55, 127

Subramaniam, A. 2004, ApJ, 604, L41

Tifft, W. G., \& Snell, C. M. 1971, MNRAS, 151, 365

Vallenari, A., Chiosi, C., Bertelli, G., \& Ortolani, S. 1996, A\&A, 309, 358

Vallenari, A., Chiosi, C., Bertelli, G., et al. 1996, A\&A, 309, 367

van der Marel, R. P. 2001, AJ, 122, 1827

van der Marel, R. P., \& Cioni, M.-R. L. 2001, AJ, 122, 1807

van den Bergh, S. 1999, in The Galaxies of the Local Group, Cambridge Astrophysics Series, 35

van den Bergh, S. 1998, ApJ, 507, 39

Westerlund, B. E. 1997, in The Magellanic Clouds, Cambridge Astrophysics Series, 29

Wood, P. R., Bessel, M. S., \& Paltoglou, G. 1985, ApJ, 290, 477 